\title{
Stability results for impulsive functional differential equations with infinite delay
}

\author{
Teresa Faria*, Marta C. Gadotti ${ }^{\dagger}$, and José J. Oliveira ${ }^{\ddagger}$
}

$\left(^{*}\right)$ Departamento de Matemática and CMAF, Faculdade de Ciências, Universidade de Lisboa, Campo Grande, 1749-016 Lisboa, Portugal e-mail: tfaria@ptmat.fc.ul.pt

$\left(^{\dagger}\right)$ Departamento de Matemática, IGCE - Universidade Estadual Paulista, Avenida 24A 1515 13506-700 Rio Claro SP, Brazil e-mail: martacg@rc.unesp.br

$\left(^{\ddagger}\right)$ Departamento de Matemática e Aplicações and CMAT, Escola de Ciências, Universidade do Minho, Campus de Gualtar, 4710-057 Braga, Portugal e-mail: jjoliveira@math.uminho.pt

\begin{abstract}
For a family of differential equations with infinitive delay and impulses, we establish conditions for the existence of global solutions and for the global asymptotic and global exponential stabilities of an equilibrium point. The results are used to give stability criteria for a very broad family of impulsive neural network models with both unbounded distributed delays and bounded time-varying discrete delays. Most of the impulsive neural network models with delay recently studied are included in the general framework presented here.
\end{abstract}

Keywords: infinite delay; impulses; existence of solutions; Cohen-Grossberg neural network; global asymptotic stability; global exponential stability.

2010 Mathematics Subject Classification: 34K45, 34K25, 34K20, 92B20.

\section{Introduction}

In nature, many evolutionary systems go through momentary abrupt changes, due to sudden phenomena in the environment. In population dynamics, these short-time phenomena include weather disasters, earthquakes, harvesting, migration of birds. In many different fields, like physics, chemical technology, machinery control, biotechnology or operation research, pulse perturbations have been introduced as a tool to control and stabilize solutions to problems. When considering the entire evolutionary system of such processes, one may consider that these changes are instantaneous, so that they are introduced as impulses in the respective models.

As a result of these multiple applications, the theory of impulsive differential equations has emerged as an important area of investigation [20]. In order to have more realistic 
models, often the past history of the systems should be taken into account - which has led to the introduction of time-delays in differential equations [14]. In recent years, the stability of an equilibrium for problems involving differential equations with delay and impulses has received a great deal of attention. Namely, natural and artificial neural networks have become an important area of research since the work of Hopfield [18], in view of their multiple applications in pattern recognition, optimization, signal processing, etc. Since the synaptic transmission of information among neurons - or their artificial representation - is not instantaneous, delays have been incorporated in dynamical systems modelling neural networks, as well as impulses occurring at certain fixed times [10]. More recently, functional differential equations (FDEs) with impulses and infinite delay have been considered and used as models for neural netwoks, see [8], [21], [22], [26], [27], [32], and [34]. The stability of equilibria in neural networks is particularly important, since the stationary states represent possible optimal solutions of the system in optimization problems, or stored patterns in associative memories. Establishing the global stability of a unique equilibrium has become one the major goals when implementing an artificial network.

In this paper, we consider a system of impulsive differential equations with infinite delay given in abstract form as

$$
\begin{aligned}
& \dot{x}_{i}(t)=f_{i}\left(t, x_{t}\right), 0 \leq t \neq t_{k}, \\
& \Delta\left(x_{i}\left(t_{k}\right)\right):=x_{i}\left(t_{k}^{+}\right)-x_{i}\left(t_{k}^{-}\right)=I_{i k}\left(x_{i}\left(t_{k}^{-}\right)\right), \quad i=1,2, \ldots n, k \in \mathbb{N} .
\end{aligned}
$$

Here, $f_{i}(t, \varphi)$ are real continuous functions for $t \geq 0$ and $\varphi:(-\infty, 0] \rightarrow \mathbb{R}^{n}$ on some space of functions to be defined later, $I_{i k}: \mathbb{R} \rightarrow \mathbb{R}$ are continuous, and $\left\{t_{k}\right\}_{k \in \mathbb{N}}$ is a sequence in $(0, \infty)$ such that $t_{k} \nearrow \infty$ as $k \rightarrow \infty$. For $t \geq 0$, we define the history function $x_{t}:(-\infty, 0] \rightarrow \mathbb{R}^{n}$ by

$$
x_{t}(s)=x(t+s), \quad s \in(-\infty, 0] .
$$

To give an initial condition for (1.1) at time $t=\sigma$ is to give the past of the system for $s \leq \sigma$, i.e., to require that $x(\sigma+s)=\varphi(s)$ for $s \leq 0$, for some prescribed function $\varphi:(-\infty, 0] \rightarrow \mathbb{R}^{n}$. With the above notation, we write

$$
x_{\sigma}=\varphi \text {. }
$$

When dealing with continuous FDEs with infinite delay, the choice of an admissible Banach phase space requires special attention, in order to have well-posedness of the initial value problem and standard results on existence, uniqueness, continuation of solutions (see [12], [13], [16]). The situation is far more complicated when impulses are added. For impulsive FDEs with bounded and unbounded delay, existence, uniqueness or global continuation of solutions have been studied by some authors, see e.g. Ballinger and Liu [1, 23], Ouahab $[29,30]$ or Ye [34]. Most of the literature often refers to a phase space satisfying a certain axiomatic under which these results are achieved, however without providing either an explicit phase space, or proofs of the results. In many papers, a phase space is not even mentioned, much less the problem of such a suitable choice. For this reason, here we spend some time with a correct formulation of a phase space and the proof of existence results.

After the introduction, the present paper is divided into four sections. Section 2 is a preliminary section, where some notation and a concrete phase space for (1.1) are introduced. 
Section 3 is dedicated to proving the existence of global solutions to (1.1) in the chosen phase space. In fact, a slightly more general system will be studied, cf. system (2.1).

The main results on stability, concerning both the global asymptotic and the global exponential stabilities of a stationary solution for a class of impulsive FDEs with infinite delay, are presented in Section 4. Note that for the impulsive systems under consideration, from the results in Section 3 it follows that the solutions will be defined for all $t \geq 0$. Most studies use a type of Lyapunov functional to obtain results on global attractivity. Instead, here we use the techniques described in the works of Faria and Oliveira in [5],[6], rather than a Lyapunov functional approach.

We are especially interested in applying these results to neural networks which can be written in the general form (1.1). In Section 5, we establish criteria for stability for several impulsive generalized Cohen-Grossberg models. The advantage of our general formulation is that it applies to a broad family of impulsive FDEs with unbounded delays, which includes as particular cases most of the models analysed in the recent literature (see e.g. [9], [21], [22], [33]).

\section{Notation and preliminaries}

For a compact interval $[\alpha, \beta]$ of $\mathbb{R}$, let $P C\left([\alpha, \beta] ; \mathbb{R}^{n}\right)$ be the space of piecewise continuous functions from $[\alpha, \beta]$ to $\mathbb{R}^{n}$ and left continuous on $(\alpha, \beta], P C\left([\alpha, \beta] ; \mathbb{R}^{n}\right)=\left\{\phi:[\alpha, \beta] \rightarrow \mathbb{R}^{n} \mid \phi\right.$ is continuous everywhere except for a finite number of points $s \in[\alpha, \beta)$ for which $\phi\left(s^{-}\right), \phi\left(s^{+}\right)$ exist and $\left.\phi\left(s^{-}\right)=\phi(s)\right\}$, equipped with the supremum norm $\|\phi\|_{\infty}=\sup _{s \in[\alpha, \beta]}|\phi(s)|$, where $|\cdot|$ is a chosen norm in $\mathbb{R}^{n}$. Denote by $R\left([\alpha, \beta] ; \mathbb{R}^{n}\right)$ the closure of $P C\left([\alpha, \beta] ; \mathbb{R}^{n}\right)$ with respect to the supremum norm in the space of all bounded functions from $[\alpha, \beta]$ to $\mathbb{R}^{n}$. The space $R\left([\alpha, \beta] ; \mathbb{R}^{n}\right)$ is the space of normalized (from the left) regulated (or ruled) functions from $[\alpha, \beta]$ to $\mathbb{R}^{n}$, i.e, the space of functions $f:[\alpha, \beta] \rightarrow \mathbb{R}^{n}$ with only discontinuities of the first kind, and left continuous on $(\alpha, \beta] ; R\left([\alpha, \beta] ; \mathbb{R}^{n}\right)$ is a Banach space and every function in $R\left([\alpha, \beta] ; \mathbb{R}^{n}\right)$ has at most countably many discontinuities (see e.g. p. 146 of $[3]$ and Chapter 3 of $[17])$.

Define the space $P C=P C\left((-\infty, 0] ; \mathbb{R}^{n}\right)$ as the space of functions from $(-\infty, 0]$ to $\mathbb{R}^{n}$ for which the restriction to each compact interval $[\alpha, \beta] \subset(-\infty, 0]$ is in $R\left([\alpha, \beta] ; \mathbb{R}^{n}\right)$. Clearly, if $\phi \in P C$ then $\phi$ is continuous everywhere except at most for a enumerable number of points $s=s_{k}$, and $\phi\left(s_{k}^{-}\right), \phi\left(s_{k}^{+}\right)$exist with $\phi\left(s_{k}\right)=\phi\left(s_{k}^{-}\right)$. Denote by $B P C$ the subspace of all bounded functions in $P C, B P C=B P C\left((-\infty, 0] ; \mathbb{R}^{n}\right)=\{\phi \in P C: \phi$ is bounded on $(-\infty, 0]\}$, with the supremum norm $\|\phi\|_{\infty}=\sup _{s \leq 0}|\phi(s)|$. For $\beta \in \mathbb{R}$, in a similar way we define the spaces $P C\left((-\infty, \beta] ; \mathbb{R}^{n}\right)$ and $B P C\left((-\infty, \beta] ; \mathbb{R}^{n}\right)$.

Fix a function $g$ such that:

(g1) $g:(-\infty, 0] \rightarrow[1, \infty)$ is a non-increasing continuous function and $g(0)=1$;

(g2) $\lim _{u \rightarrow 0^{-}} \frac{g(s+u)}{g(s)}=1$ uniformly on $(-\infty, 0]$;

(g3) $g(s) \rightarrow \infty$ as $s \rightarrow-\infty$.

Note that these conditions are fulfilled for e.g. $g(s)=e^{-\alpha s}$, where $\alpha>0$. We shall consider the phase space

$$
P C_{g}=\left\{\phi \in P C: \sup _{s \leq 0} \frac{|\phi(s)|}{g(s)}<\infty\right\}
$$


with the norm

$$
\|\phi\|_{g}=\sup _{s \leq 0} \frac{|\phi(s)|}{g(s)} .
$$

It is clear that $B P C \subset P C_{g}$, with $\|\phi\|_{g} \leq\|\phi\|_{\infty}$ for $\phi \in B P C$. If $B P C$ is considered as a subspace of $P C_{g}$, we often write $B P C_{g}$. The spaces $\left(B P C,\|\cdot\|_{\infty}\right)$ and $\left(P C_{g},\|\cdot\|_{g}\right)$ are Banach spaces (see Section 3).

Let $D \subset P C_{g}, f: \mathbb{R} \times D \rightarrow \mathbb{R}^{n}, I_{k}: D \rightarrow \mathbb{R}^{n}(k \in \mathbb{N})$ be continuous functions, and $\left(t_{k}\right)_{k \in \mathbb{N}}$ a given sequence on $(0, \infty)$ such that $t_{k} \nearrow \infty$ as $k \rightarrow \infty$. Consider impulsive FDEs in $P C_{g}$ in the general form

$$
\begin{gathered}
\dot{x}(t)=f\left(t, x_{t}\right), \quad t \geq 0, t \neq t_{k} \\
\Delta x\left(t_{k}\right)=I_{k}\left(x_{t_{k}}\right), \quad k=1,2, \ldots,
\end{gathered}
$$

where $\Delta x\left(t_{k}\right)=x\left(t_{k}^{+}\right)-x\left(t_{k}^{-}\right)$. Here, if $x:(-\infty, a) \rightarrow \mathbb{R}^{n}(a \in \mathbb{R}$ or $a=\infty)$ has at most countably many jump discontinuities and $t \in(-\infty, a)$, as usual $x_{t}$ denotes the function $x_{t}:(-\infty, 0] \rightarrow \mathbb{R}^{n}$ defined by $x_{t}(s)=x(t+s), s \leq 0$. For the equation with impulses (2.1), we shall always consider initial conditions in $B P C, x_{\sigma}=\phi \in B P C$. Alternatively, we may choose $B P C$ as the phase space for (2.1), where now $D \subset B P C$, and $f: \mathbb{R} \times D \rightarrow \mathbb{R}^{n}, I_{k}$ : $D \rightarrow \mathbb{R}^{n}(k \in \mathbb{R})$ are continuous functions relative to the norm $\|\cdot\|_{\infty}$ in $B P C$.

Consider (2.1) and BPC as the set of admissible initial conditions.

Definition 2.1. A function $x(t)$ is called a solution of system (2.1) corresponding to $(\sigma, \phi)$ if there is $d>\sigma$ such that $x:(-\infty, d] \rightarrow \mathbb{R}^{n}$ is continuous for $t \in[\sigma, d] \backslash\left\{t_{k}: k \in \mathbb{N}\right\}, x\left(t_{k}^{-}\right)$ and $x\left(t_{k}^{+}\right)$exist with $x\left(t_{k}\right)=x\left(t_{k}^{-}\right)$for $t_{k} \in[\sigma, d](k \in \mathbb{N})$, the derivative of $x(t)$ exists for $t \in[\sigma, d] \backslash\left\{t_{k}: k \in \mathbb{N}\right\}, x(t)$ satisfies system (2.1), and $x_{\sigma}=\phi$.

In order to simplify the notation, in general we shall take $f$ in (2.1) defined in the whole space (i.e, either $D=P C_{g}$ or $D=B P C$ ) and initial conditions will be given at $t=0$ :

$$
x_{0}=\phi, \quad \phi \in B P C .
$$

For differential equations with impulses and unbounded delay, one should take some care with the choice of a suitable phase space and set of initial conditions. Even for the case without impulses, dealing with FDEs with unbounded delay requires a careful abstract formulation of an admissible phase space. For $g$ satisfying (g1)-(g3), the space $U C_{g}$ defined by

$$
U C_{g}=\left\{\varphi \in C\left((-\infty, 0], \mathbb{R}^{n}\right): \sup _{\theta \leq 0} \frac{|\varphi(\theta)|}{g(\theta)}<\infty, \frac{\varphi(\theta)}{g(\theta)} \text { is uniformly continuous on }(-\infty, 0]\right\}
$$

with the norm $\|\cdot\|_{g}$ is an admissible phase space in the sense of [13], [16]. This means that the normed space $\mathcal{B}=U C_{g}$ satisfies the axiomatic conditions for an admissible space given below:

(A) There are a constant $J>0$ and functions $K, M:[0, \infty) \rightarrow[0, \infty)$, with $K$ continuous and $M$ locally bounded, such that the following conditions hold:

If $x:(-\infty, d] \rightarrow \mathbb{R}^{n}, d>a$, is such that $x_{a} \in \mathcal{B}$ and $\left.x\right|_{[a, d]} \in C\left([a, d] ; \mathbb{R}^{n}\right)$, then for all $t \in[a, d]:$ 
(i) $x_{t} \in \mathcal{B}$;

(ii) $|x(t)| \leq J\left\|x_{t}\right\|_{\mathcal{B}}$;

(iii) $\left\|x_{t}\right\|_{\mathcal{B}} \leq K(t-a) \sup \{|x(s)|: a \leq s \leq t\}+M(t-a)\left\|x_{a}\right\|_{\mathcal{B}}$;

(iv) for $t \in[a, d], t \mapsto x_{t} \in \mathcal{B}$ is a continuous function.

(B) $\mathcal{B}$ is complete.

Since (A)-(B) are satisfied, for an abstract FDE in the phase space $U C_{g}$,

$$
\dot{x}(t)=f\left(t, x_{t}\right),
$$

where $f: D \subset \mathbb{R} \times U C_{g} \rightarrow \mathbb{R}^{n}$ is continuous, the standard existence and uniqueness type results hold ([13],[16]). Moreover, $U C_{g}$ is a fading memory space, and therefore precompactness results for bounded positive orbits in $U C_{g}$ are valid ([12]). Clearly, results for the local existence and uniqueness of solution for the initial value problem (2.1)-(2.2) follow from the corresponding results for DDEs without impulses (2.3).

As regards the existence of global solutions for impulsive FDEs with infinite delay, we refer the reader to [15], [30], [34], also for further references. Nevertheless, the study of continuation of solutions for the impulsive system with unbounded delay (2.1) seems not to have been carried out consistently. Most of the times authors either assume that all solutions with admissible initial conditions are defined for all $t \geq 0$, or introduce axiomatically a phase space $\mathcal{B}$, which should be complete and a 'fading' memory space; this means that $\mathcal{B}$ should satisfy axioms $(\mathrm{A})(\mathrm{i})$-(iii) and (B), with $C\left([a, d] ; \mathbb{R}^{n}\right)$ replaced by $P C\left([a, d] ; \mathbb{R}^{n}\right)$ - note however that hypothesis (A)(iv) fails for FDEs with impulses. Frequently, either an explicit space is not given, or the above axioms are not verified for a given one. In many papers, a phase space is not even mentioned. This situation motivates us to establish sufficient conditions for the existence of global solutions to (2.1) in the concrete phase space $P C_{g}$. Spaces $P C_{g}$ will be used throughout Sections 4 and 5 , however other concrete suitable phase spaces will be mentioned in Section 3 .

In a framework assuring global continuation of all admissible solutions to (2.1), we will be concerned with the stability of an equilibrium solution, if it exists. For $x^{*}=\left(x_{1}^{*}, \ldots, x_{n}^{*}\right) \in$ $\mathbb{R}^{n}$, we also use $x^{*}$ to denote the constant function $x(t)=x^{*}$ for $t$ on an interval of $\mathbb{R}$. We say that $x^{*}$ is an equilibrium of $(2.1)$ if $f\left(t, x^{*}\right)=0$ and $I_{k}\left(x^{*}\right)=0$ for all $k \in \mathbb{N}$.

Definition 2.2. Consider the phase space $\mathcal{D}$ with norm $\|\cdot\|$, where either $(\mathcal{D},\|\cdot\|)=$ $\left(P C_{g},\|\cdot\|_{g}\right)$ for some $g$ satisfying $(g 1)-(g 3)$, or $(\mathcal{D},\|\cdot\|)=\left(B P C,\|\cdot\|_{\infty}\right)$, and take BPC as the set of admissible initial conditions. Suppose that $x^{*}$ is an equilibrium of (2.1). The equilibrium $x^{*}$ is said to be: (i) stable if for any $\sigma>0$ and $\varepsilon>0$ there is $\delta=\delta(\sigma, \varepsilon)>0$ such that $\left\|x_{t}(\sigma, \phi)-x^{*}\right\|<\varepsilon$ for all $\phi \in B P C$, with $\left\|\phi-x^{*}\right\|<\delta$ and $t \geq \sigma$; (ii) uniformly stable if $\delta$ in (i) does not depend on $\sigma$; (iii) globally asymptotically stable if $x^{*}$ is stable and globally attractive in $\mathbb{R}^{n}$, i.e., $x(t) \rightarrow x^{*}$ as $t \rightarrow \infty$, for all solutions $x(t)$ with initial condition in BPC; (iv) globally exponentially stable if there are positive constants $\varepsilon$, $M$ such that

$$
\left|x(t, 0, \phi)-x^{*}\right| \leq M e^{-\varepsilon t}\left\|\phi-x^{*}\right\|_{\infty} \quad \text { for } \quad t \geq 0, \phi \in B P C .
$$




\section{Existence of solutions}

Although we take $B P C$ as the set of admissible initial conditions for an FDE with impulses, this space is not big enough for the purpose of proving the global attractivity of an equilibrium of (2.1). Rather than $B P C$, we shall work on a space $P C_{g}$ where $g$ satisfies (g1)-(g3). It is well known that the spaces $R\left([\alpha, \beta] ; \mathbb{R}^{n}\right)(\alpha, \beta \in \mathbb{R}, \alpha<\beta)$ equipped with the norm $\|\cdot\|_{\infty}$ are Banach spaces, hence $B P C$ is also a Banach space with $\|\cdot\|_{\infty}$. Now, it is easy to show that:

Lemma 3.1. $P C_{g}$ with a Banach space with $\|\cdot\|_{g}$.

Proof. Let $\left(\varphi_{n}\right)$ be a Cauchy sequence in $P C_{g}$, and fix any $\varepsilon>0$. There is $n_{0} \in \mathbb{N}$ such that for all $n>n_{0}$ and $p \in \mathbb{N}$

$$
\left\|\varphi_{n+p}-\varphi_{n}\right\|_{g}=\sup _{s \leq 0} \frac{1}{g(s)}\left|\varphi_{n+p}(s)-\varphi_{n}(s)\right|<\varepsilon .
$$

For each $s \leq 0$, from the above inequality it follows that the sequence $\varphi_{n}(s)$ is a Cauchy sequence in $\mathbb{R}^{n} ;$ moreover, for its limit $\varphi(s):=\lim \varphi_{n}(s)$, we get

$$
\frac{1}{g(s)}\left|\varphi_{n}(s)-\varphi(s)\right| \leq \varepsilon
$$

for all $n>n_{0}$. Consequently, $\sup _{s \leq 0} \frac{1}{g(s)}\left|\varphi_{n}(s)-\varphi(s)\right| \rightarrow 0$ as $n \rightarrow \infty$. On the other hand, for each compact interval $[\alpha, \beta] \subset(-\infty, 0], \varphi_{\left.n\right|_{[\alpha, \beta]}} \rightarrow \varphi_{\left.\right|_{[\alpha, \beta]}}$ uniformly in $[\alpha, \beta]$. Since the spaces $R\left([\alpha, \beta] ; \mathbb{R}^{n}\right)$ equipped with the norm $\|\cdot\|_{\infty}$ are complete, $\varphi_{\left.\right|_{[\alpha, \beta]}} \in R\left([\alpha, \beta] ; \mathbb{R}^{n}\right)$ for each $[\alpha, \beta] \subset(-\infty, 0]$. Therefore $\varphi \in P C_{g}$ and $\left\|\varphi_{n}-\varphi\right\|_{g} \rightarrow 0$ as $n \rightarrow \infty$.

We return to the space $B P C$ equipped with either the norm of uniform convergence $\|\cdot\|_{\infty}$ or the norm $\|\cdot\|_{g}$ where $g$ satisfies (g1)-(g3). The norm in $B P C$ will be denoted simply by $\|\cdot\|$. For a given $x \in B P C\left((-\infty, b] ; \mathbb{R}^{n}\right)$ where $b>0$, clearly the function $\Phi:[0, b] \rightarrow B P C$, $t \mapsto x_{t}=: \Phi(t)$ is not continuous, unless $x$ is itself continuous: in fact, $\Phi$ is discontinuous at any $t \in[0, b]$ such that $x$ has a jump discontinuity for some $t_{0} \in(-\infty, t)$. In order to have continuity of the map $\Phi$, one has to consider a suitable norm in $B P C$, see Lemma 3.2 below. Nevertheless, in general the function $t \mapsto f\left(t, x_{t}\right)$ is measurable on intervals $[0, b]$, and therefore summable on $[0, b]$ provided it is dominated by some summable function.

Consider the impulsive FDE in $B P C$

$$
\begin{aligned}
& \dot{x}(t)=f\left(t, x_{t}\right), \quad t \geq 0, t \neq t_{k} \\
& \Delta x\left(t_{k}\right)=I_{k}\left(x_{t_{k}}\right), \quad k=1,2,
\end{aligned}
$$

where $0<t_{1}<t_{2}<\ldots$ with $t_{k} \rightarrow \infty$, and the functions $f:[0, \infty) \times B P C \rightarrow \mathbb{R}^{n}, I_{k}$ : $B P C \rightarrow \mathbb{R}^{n}$ are continuous, $k \in \mathbb{N}$, subject to bounded initial conditions:

$$
x_{0}=\phi, \quad \phi \in B P C \text {. }
$$

Under some conditions, we prove the existence of global solutions for the problem (3.1)(3.2), following the approach in $[29,30]$. The purpose here is not to give optimal sufficient conditions for global solutions to exist (cf. Remark 3.1), but simply to provide a sufficiently general setting which covers the impulsive systems studied in Sections 4 and 5. As usual, a fixed point argument is used, in this case the Leray-Schauder alternative theorem (see [11], p. 124): 
Theorem 3.1. Let $C$ be a convex subset of a normed space $E$, and assume that $0 \in C$. If $F: C \rightarrow C$ is a completely continuous operator, then either the set $\{x \in C: x=\lambda F x$ for some $\lambda \in(0,1)\}$ is unbounded or $F$ has a fixed point.

Below, if $b=\infty$, the interval $[0, b]$ means $[0, \infty)$.

Theorem 3.2. Consider BPC with the norm $\|\cdot\|$, where $\|\cdot\|=\|\cdot\|_{\infty}$ or $\|\cdot\|=\|\cdot\|_{g}$. For $b>0$ or $b=\infty$, assume that:

(h1) for all $x \in B P C\left((-\infty, b] ; \mathbb{R}^{n}\right)$, the function $t \mapsto f\left(t, x_{t}\right)$ is measurable on $[0, b]$;

(h2) there are a locally integrable function $p:[0, b] \rightarrow[0, \infty)$ and a continuous nondecreasing function $q:[0, \infty) \rightarrow[0, \infty)$ with $q(u)>0$ for $u>0$ and such that $\int^{\infty} \frac{d u}{q(u)}=\infty$, satisfying

$$
|f(t, \psi)| \leq p(t) q(\|\psi\|), \quad \text { for } \quad t \in[0, b], \psi \in B P C ;
$$

(h3) for each $k \in \mathbb{N}, I_{k}$ maps bounded sets of BPC into bounded sets of $\mathbb{R}^{n}$.

Then, for each $\phi \in B P C$, the problem (3.1)-(3.2) has a solution $x(t)$ defined on $[0, b]$.

Proof. We suppose that $B P C$ is equipped with the norm of uniform convergence. For $\|\cdot\|=\|\cdot\|_{g}$, the proof follows in a similar way. Let $\phi \in B P C$ be given and fix $\bar{b}>0$ with $\bar{b}=b$ if $b \in \mathbb{R}$ and $\bar{b}<\infty$ if $b=\infty$. We prove that there exists a solution $x(t)$ of the problem (3.1)-(3.2) defined for $t \leq \bar{b}$. For simplicity, in what follows we write $b$ instead of $\bar{b}$.

Set $t_{0}=0$, and write $b \in\left(t_{n-1}, t_{n}\right]$ for some $n \in \mathbb{N}$. We have $\left(\int_{0}^{t} f\left(s, x_{s}\right) d s\right)^{\prime}=f\left(t, x_{t}\right)$ on $[0, b]$ for $x \in B P C\left((-\infty, b] ; \mathbb{R}^{n}\right)$, and consequently $x(t)$ is a solution of (3.1)-(3.2) on $[0, b]$ if and only if $x(t)=(N x)(t)$ for $t \leq b$, where $N: X \rightarrow X$ is the operator defined on $X:=\left\{x:(-\infty, b] \rightarrow \mathbb{R}^{n} \mid x_{0}=\phi, x\left(0^{+}\right)=\phi(0)\right.$ and $\left.x_{\mid[0, b]} \in P C\left([0, b] ; \mathbb{R}^{n}\right)\right\}$ by

$$
(N x)(t)=\left\{\begin{array}{l}
\phi(t), \quad t \leq 0 . \\
\phi(0)+\int_{0}^{t} f\left(s, x_{s}\right) d s+\sum_{k: 0<t_{k}<t} I_{k}\left(x_{t_{k}}\right), \quad 0 \leq t \leq b, x \in X .
\end{array}\right.
$$

For $s \leq b$, write $x_{s}=\tilde{\phi}_{s}+\bar{x}_{s}$, where $\tilde{\phi}(s)=\phi(s)$ for $s \leq 0$ and $\tilde{\phi}(s)=\phi(0)$ for $0 \leq s \leq b$. Then, $N x=x$ if and only if $N_{0} \bar{x}=\bar{x}$, where, after dropping the bars for simplicity, $N_{0}: X_{0} \rightarrow X_{0}$ is the operator given by

$$
\left(N_{0} x\right)(t)=0 \text { for } t \leq 0,\left(N_{0} x\right)(t)=\int_{0}^{t} f\left(s, \tilde{\phi}_{s}+x_{s}\right) d s+\sum_{k: 0<t_{k}<t} I_{k}\left(\tilde{\phi}_{t_{k}}+x_{t_{k}}\right) \text { for } 0 \leq t \leq b,
$$

and $X_{0}$ is the space $X_{0}:=\left\{x \in B P C\left((-\infty, b] ; \mathbb{R}^{n}\right): x_{0}=0, x\left(0^{+}\right)=0\right\}$, which can be identified with the subspace of the functions $x(t)$ in $P C\left([0, b] ; \mathbb{R}^{n}\right)$ with $x\left(0^{+}\right)=0$.

Step 1. $N_{0}$ is completely continuous.

Let $\left\{x_{m}\right\} \subset X_{0}$ and $x_{m} \rightarrow x$ uniformly on $[0, b]$. Then,

$\left\|N_{0} x_{m}-N_{0} x\right\|_{\infty} \leq \int_{0}^{b}\left|f\left(s, \tilde{\phi}_{s}+\left(x_{m}\right)_{s}\right)-f\left(s, \tilde{\phi}_{s}+x_{s}\right)\right| d s+\sum_{1 \leq k \leq n}\left|I_{k}\left(\tilde{\phi}_{t_{k}}+\left(x_{m}\right)_{t_{k}}\right)-I_{k}\left(\tilde{\phi}_{t_{k}}+x_{t_{k}}\right)\right|$.

From the continuity of the functions $f$ and $I_{k},(\mathrm{~h} 2)$ and Lebesgue's dominated convergence theorem, we get $\left\|N_{0} x_{m}-N_{0} x\right\|_{\infty} \rightarrow 0$. 
Since $\left\|N_{0} x\right\|_{\infty} \leq \int_{0}^{b} p(s) q\left(\left\|\tilde{\phi}_{s}+x_{s}\right\|_{\infty}\right) d s+\sum_{1 \leq k \leq n}\left|I_{k}\left(\tilde{\phi}_{s}+x_{t_{k}}\right)\right|$, then $N_{0}$ maps bounded sets of $X_{0}$ into bounded sets of $X_{0}$.

Next, write $N_{0}=N_{1}+N_{2}$, where $N_{1}, N_{2}: X_{0} \rightarrow X_{0}$ are the operators given by $\left(N_{i} x\right)(t)=$ 0 for $t \leq 0$ and

$$
\left(N_{1} x\right)(t)=\int_{0}^{t} f\left(s, \tilde{\phi}_{s}+x_{s}\right) d s, \quad\left(N_{2} x\right)(t)=\sum_{k: 0<t_{k}<t} I_{k}\left(\tilde{\phi}_{s}+x_{t_{k}}\right) \quad \text { for } 0 \leq t \leq b .
$$

Fix $M>0$. For any $\tau_{0}, \tau \in[0, b]$, and $x \in X_{0}$ such that $\|x\|_{\infty} \leq M$, we have $\left|\left(N_{1} x\right)(\tau)-\left(N_{1} x\right)\left(\tau_{0}\right)\right| \leq q\left(\|\phi\|_{\infty}+M\right) \int_{\tau_{0}}^{\tau} p(s) d s$, which converges uniformly to zero as $\tau \rightarrow \tau_{0}$, independently of $x$. This means that $N_{1}$ maps a ball $B_{M}(0)$ of $X_{0}$ into an equicontinuous set of the space $\left\{x:(-\infty, b] \rightarrow \mathbb{R}^{n}: x\right.$ is continuous and $\left.x_{0}=0\right\}$, which can be identified with the subspace of the functions in $C\left([0, b] ; \mathbb{R}^{n}\right)$ with $x(0)=0$. From Ascoli-Arzelà theorem, we conclude that $N_{1}$ is completely continuous. On the other hand, hypothesis (h3) means that $I_{k}$ are completely continuous, and therefore the same happens to the operator $\mathrm{N}_{2}$.

Step 2. The set $\mathcal{E}=\left\{x \in X_{0}: x=\lambda N_{0} x\right.$ for some $\left.\lambda \in(0,1)\right\}$ is bounded.

Let $\lambda \in(0,1)$ and $x=\lambda N_{0} x$. For $t \in\left[0, \bar{t}_{1}\right]$ where $\bar{t}_{1}=\min \left\{t_{1}, b\right\}$, we have

$$
\left\|x_{t}\right\|_{\infty} \leq \lambda \int_{0}^{t} p(s) q\left(\left\|\tilde{\phi}_{s}+x_{s}\right\|_{\infty}\right) d s \leq \int_{0}^{t} p(s) q\left(\left\|\tilde{\phi}_{s}+x_{s}\right\|_{\infty}\right) d s=: \beta(t) .
$$

The function $\beta(t)$ is non-decreasing and $\beta(0)=0$; since $q$ is non-decreasing, it also satisfies the differential inequality $\beta^{\prime}(t) \leq p(t) q\left(\|\phi\|_{\infty}+\beta(t)\right)$. We now use the well-known Osgood's test argument: if the solution $u(t)$ of the initial value problem $u(0)=0, u^{\prime}(t)=p(t) q\left(\|\phi\|_{\infty}+\right.$ $u(t))$ is not bounded on $[0, \tau)$ with $\tau \leq \bar{t}_{1}$, then $u(t) \rightarrow \infty$ as $t \rightarrow \tau^{-}$and

$$
\int_{0}^{\tau} p(t) d t=\int_{0}^{\tau} \frac{u^{\prime}(t)}{q\left(\|\phi\|_{\infty}+u(t)\right)} d t=\int_{\|\phi\|_{\infty}}^{\infty} \frac{d u}{q(u)}=\infty
$$

a contradiction. This implies that there is $C_{1}>0$ such that $\beta(t) \leq C_{1}$ on $\left[0, \bar{t}_{1}\right]$, where $C_{1}$ may dependent on $\phi$ but not on $x$. Hence, $\left\|x_{t}\right\|_{\infty} \leq C_{1}$ for all $t \in\left[0, \bar{t}_{1}\right]$.

Now, suppose that $b>t_{1}$. From (h3), consider $c_{1}>0$ such that $\left|I_{1}(y)\right| \leq c_{1}$ for all $y \in B C P$ with $\|y\|_{\infty} \leq C_{1}+\|\phi\|_{\infty}$. For $t \in\left(t_{1}, \bar{t}_{2}\right]$ where $\bar{t}_{2}=\min \left\{b, t_{2}\right\}$, we obtain

$$
\left\|x_{t}\right\|_{\infty} \leq \int_{0}^{t} p(s) q\left(\left\|\tilde{\phi}_{s}+x_{s}\right\|_{\infty}\right) d s+c_{1}
$$

Proceeding as above, where now $\beta(t)=c_{1}+\int_{0}^{t} p(s) q\left(\left\|\tilde{\phi}_{s}+x_{s}\right\|_{\infty}\right) d s$ for $t \in\left[0, \bar{t}_{2}\right]$, in a similar way we deduce that there is a constant $C_{2}$ such that $\left\|x_{t}\right\|_{\infty} \leq \beta(t) \leq C_{2}$ for all $t \in\left[0, \bar{t}_{2}\right]$. By induction, we conclude that there is $C>0$ such that $\left\|x_{t}\right\|_{\infty} \leq C$ for all $t \in[0, b]$, hence the set $\mathcal{E}$ is bounded.

Thus, from Theorem 3.1 there is a fixed point of $N$, and the proof is complete.

Clearly (3.3) holds if $|f(t, \psi)|$ has a sublinear-type growth as described in the following criterion: 
Corollary 3.1. Let BPC be equipped with either the norm $\|\cdot\|=\|\cdot\|_{\infty}$ or $\|\cdot\|=\|\cdot\|_{g}$. Assume (h1), (h3), and that for each $b>0$ there are integrable functions $p, q:[0, b], \rightarrow[0, \infty)$ such that

$$
|f(t, \psi)| \leq p(t)+q(t)\|\psi\|, \quad \text { for all } t \in[0, b], \psi \in B P C .
$$

Then, for each $\phi \in B P C$, the problem (3.1)-(3.2) has a solution $x(t)$ defined for $t \geq 0$.

Remark 3.1. As for FDEs without impulses, in Theorem 3.2 it is actually sufficient to require that $f(t, \psi)$ satisfies the Carathéodory conditions, instead of the continuity of the function $f(t, \psi)$ on both variables (see e.g. [14] p. 58, and [29, 30]). On the other hand, if the impulse functions $I_{k}$ are uniformly bounded by constants $c_{k}>0$, for each given initial condition $\phi \in B C P$ we may replace condition $\int^{\infty} \frac{d u}{q(u)}=\infty$ in (h2) by

$$
\int_{0}^{t_{n}+1} p(s) d s<\int_{M_{n}+\|\phi\|_{\infty}}^{\infty} \frac{d u}{q(u)} \text { for all } n \in \mathbb{N}, \quad \text { where } M_{n}=\sum_{k=1}^{n} c_{k},
$$

and deduce the existence of a global solution of (3.1)-(3.2). This is proven by applying in Step 2 of the above proof the argument used for the Osgood's test in each interval $\left[0, t_{1}\right],\left[t_{1}, t_{2}\right]$, etc. However, the setting in Theorem 3.2 is general enough for our purposes, since the aim is to study impulsive neural network in the form (4.1).

As mentioned previously, the impulsive nature of system (3.1) brings difficulties when working with delayed differential equations, even for the case of finite discrete delays, due to the fact that the map $t \mapsto x_{t}$ is not continuous if $x$ has at least a simple jump discontinuity. Together with the subtle problem of working with infinite delay, this makes the choice of a suitable phase space, for which the standard results on existence, uniqueness, continuation of solutions should be valid, a difficult task. As we shall see in Sections 4 and 5 , in terms of applications it is very useful to use the norm $\|\cdot\|_{g}\left(\right.$ or $\left.\|\cdot\|_{\infty}\right)$ in $B P C$, but in fact a more efficient norm is defined below.

For $g$ satisfying (g1)-(g3), define the space $L_{g}^{1}=L_{g}^{1}\left((-\infty, 0] ; \mathbb{R}^{n}\right)$ as

$$
L_{g}^{1}=\left\{\varphi:(-\infty, 0] \rightarrow \mathbb{R}^{n} \mid \frac{\varphi(s)}{g(s)} \in L^{1}\left((-\infty, 0] ; \mathbb{R}^{n}\right)\right\}
$$

with the norm

$$
\|\varphi\|_{L_{g}^{1}}=\int_{-\infty}^{0} \frac{|\varphi(s)|}{g(s)} d s .
$$

The space $L_{g}^{1}\left((-\infty, b] ; \mathbb{R}^{n}\right)$ is defined in a similar way. We further assume

(g4) $g^{-1} \in L^{1}((-\infty, 0] ; \mathbb{R})$, where $g^{-1}(s)=1 / g(s)$.

Clearly, if $\varphi \in B P C$ then $\varphi \in L_{g}^{1}$, with $\|\varphi\|_{L_{g}^{1}} \leq\left\|g^{-1}\right\|_{L^{1}}\|\varphi\|_{\infty}$; and $L_{g}^{1} \cap P C \subset P C_{g}$, however this latter inclusion is not continuous for $L_{g}^{1} \cap P C$ endowed with $\|\cdot\|_{L_{g}^{1}}$.

Lemma 3.2. Let $g$ satisfy (g1)-(g4). For a given $x \in B P C\left((-\infty, b] ; \mathbb{R}^{n}\right)$ where $b>0$, the function $\Phi:[0, b] \rightarrow L_{g}^{1}, t \mapsto x_{t}$, is continuous.

Proof. Let $\left\{s_{k}\right\}$ be the set of discontinuity points for $x$. For $t_{n}, t_{0} \in[0, b]$ with $t_{n} \rightarrow t_{0}$, $\left\|x_{t_{n}}-x_{t_{0}}\right\|_{L_{g}^{1}}=\int_{-\infty}^{0} h_{n}(s) d s$, where $h_{n}(s):=\left|x\left(t_{n}+s\right)-x\left(t_{0}+s\right)\right| / g(s) \rightarrow 0$ as $n \rightarrow \infty$ for every $s \in(-\infty, 0], s \neq s_{k}-t_{0}$, and $\left|h_{n}(s)\right| \leq 2\|x\|_{\infty} / g(s) \in L^{1}$. Hence $\left\|x_{t_{n}}-x_{t_{0}}\right\|_{L_{g}^{1}} \rightarrow 0$. 
If $f:[0, \infty) \times\left(B P C,\|\cdot\|_{L_{g}^{1}}\right) \rightarrow \mathbb{R}^{n}$ is continuous, then the map from $[0, b]$ to $\mathbb{R}^{n}, t \mapsto$ $f\left(t, x_{t}\right)$, is continuous. An existence result similar to Theorem 3.2 is valid for the norm $\|\cdot\|_{L_{g}^{1}}$, as follows:

Theorem 3.3. Consider BPC with a norm $\|\cdot\|_{L_{g}^{1}}$, where g satisfies (g1)-(g4). For $b>0$ or $b=\infty$, assume that:

(i) there are a locally integrable function $p:[0, b] \rightarrow[0, \infty)$ and a continuous nondecreasing function $q:[0, \infty) \rightarrow[0, \infty)$ with $q(u)>0$ for $u>0$ and such that $\int^{\infty} \frac{d u}{q(u)}=\infty$, satisfying

$$
|f(t, \psi)| \leq p(t) q\left(\|\psi\|_{L_{g}^{1}}\right), \quad \text { for all } \quad t \in[0, b], \psi \in B P C ;
$$

(ii) for each $k \in \mathbb{N}, I_{k}$ maps bounded sets of BPC into bounded sets of $\mathbb{R}^{n}$.

Then, for each $\phi \in B P C$, the problem (3.1)-(3.2) has a solution $x(t)$ defined on $[0, b]$.

Proof. The aim is to use Theorem 3.1, to guarantee the existence of a fixed point to the operator $N: X \rightarrow X$ given by (3.4), where now $X:=\left\{x:(-\infty, b] \rightarrow \mathbb{R}^{n} \mid x_{0}=\phi, x\left(0^{+}\right)=\right.$ $\phi(0)$ and $\left.x_{\mid[0, b]} \in L^{1}\left([0, b] ; \mathbb{R}^{n}\right)\right\}$ is considered as a subset of $L_{g}^{1}\left((-\infty, b] ; \mathbb{R}^{n}\right)$. The proof follows along the lines of the proof of Theorem 3.2, with the necessary adaptations for $\|\cdot\|_{L_{g}^{1}}$ (instead of $\|\cdot\|_{\infty}$ ), and where instead of the Ascoli-Arzelà theorem we use the following characterization of compactness in $L^{1}[0, b]$ (cf. [4]): a set $K \subset L^{1}[0, b]$ is relatively compact if and only if $\int_{0}^{b}|y(t+u)-y(t)| d t \rightarrow 0$ uniformly for $y \in K$. Details are omitted.

Remark 3.2. Due to the continuity of the map $t \mapsto x_{t} \in L_{g}^{1}$, in fact other nice properties can be derived in the phase space $L_{g}^{1}$, such as pre-compactness of bounded positive orbits, however such study is behond the purposes of the present paper.

\section{Main Results on Stability}

In this section, sufficient conditions for the existence, global asymptotic stability and global exponential stability of an equilibrium point for (1.1) (???ou será (4.1)??) will be established.

We start with an auxiliary lemma.

Lemma 4.1. Suppose that $\mathbb{R}^{n}$ is equipped with the maximum norm, and assume the following hypotheses:

(H1) for all $t \geq 0$ and $\varphi \in P C_{g}$ such that $\frac{1}{g(\theta)}|\varphi(\theta)|<|\varphi(0)|$, for all $\theta<0$, then $f_{i}(t, \varphi) \varphi_{i}(0)<0$ for some $i \in\{1, \ldots, n\}$ such that $|\varphi(0)|=\left|\varphi_{i}(0)\right|$;

(H2) for each function $\widehat{I}_{k}(u):=I_{k}(u)+u\left(u \in \mathbb{R}^{n}\right)$, there is $\xi_{k}>0$ such that $\left|\widehat{I}_{k}(u)\right| \leq \xi_{k}|u|$ and $\prod_{k=1}^{\infty} \max \left\{1, \xi_{k}\right\}<\infty$.

If $x(t, 0, \varphi)$ is a solution of (1.1)-(1.2) defined on $\mathbb{R}$, then $x(t, 0, \varphi)$ is bounded and

$$
|x(t, 0, \varphi)| \leq\|\varphi\|_{g} \prod_{k=1}^{\infty} \max \left\{1, \xi_{k}\right\} .
$$


Proof. If $x(t, 0, \varphi)$ is the solution of (1.1)-(1.2), then $x(t, 0, \varphi)$ is a continuous function for $t \in\left(0, t_{1}\right)$. From Lemma 2.1 in [6], we obtain

$$
|x(t, 0, \varphi)| \leq\|\varphi\|_{g}, \quad \forall t \in\left(0, t_{1}\right] .
$$

By using (H2), for $t=t_{1}$

$$
\left|x\left(t_{1}^{+}\right)\right|=\left|\widehat{I}_{1}\left(x\left(t_{1}\right)\right)\right| \leq \xi_{1}\left|x\left(t_{1}\right)\right| \leq \xi_{1}\|\varphi\|_{g},
$$

and we get

$$
\left|x\left(t^{+}, 0, \varphi\right)\right| \leq\|\varphi\|_{g} \max \left\{1, \xi_{1}\right\}, \quad t \in\left(0, t_{1}\right] .
$$

For $t \in\left(t_{1}, t_{2}\right]$ and using the same arguments, we have

$$
|x(t, 0, \varphi)| \leq\|\varphi\|_{g} \max \left\{1, \xi_{1}\right\}, \quad t \in\left(t_{1}, t_{2}\right] .
$$

Again by using (H2), for $t=t_{2}$ we obtain

$$
\left|x\left(t_{2}^{+}\right)\right|=\left|\widehat{I}_{2}\left(x\left(t_{2}\right)\right)\right| \leq \xi_{2}\left|x\left(t_{2}\right)\right| \leq \xi_{2}\|\varphi\|_{g} \max \left\{1, \xi_{1}\right\} .
$$

Therefore,

$$
\left|x\left(t^{+}, 0, \varphi\right)\right| \leq\|\varphi\|_{g} \max \left\{1, \xi_{1}\right\} \max \left\{1, \xi_{2}\right\}, \quad t \in\left(0, t_{2}\right] .
$$

Repeating the above procedure, for $t \in\left(0, t_{l}\right], l \in \mathbb{N}$, we conclude that

$$
\left|x\left(t^{+}, 0, \varphi\right)\right| \leq\|\varphi\|_{g} \max \left\{1, \xi_{1}\right\} \ldots \max \left\{1, \xi_{l}\right\}, \quad t \in\left(0, t_{l}\right] .
$$

Consequently,

$$
|x(t, 0, \varphi)| \leq\|\varphi\|_{g} \prod_{k=1}^{\infty} \max \left\{1, \xi_{k}\right\}, \quad t \geq 0
$$

Remark 4.1. A similar estimate is obtained using the norm $\|\cdot\|_{\infty}$ in $B P C$ (cf. Lemma 2.2 in [6]). In fact, if (H1) is replaced by

(H1') for all $t \geq 0$ and $\varphi \in B P C$ such that $|\varphi(\theta)|<|\varphi(0)|$, for all $\theta<0$, then $f_{i}(t, \varphi) \varphi_{i}(0)<0$ for some $i \in\{1, \ldots, n\}$ such that $|\varphi(0)|=\left|\varphi_{i}(0)\right|$,

then in the above lemma we obtain the estimate $|x(t, 0, \varphi)| \leq\|\varphi\|_{\infty} \prod_{k=1}^{\infty} \max \left\{1, \xi_{k}\right\}$.

We now consider the following non-autonomous impulsive system:

$$
\begin{aligned}
& \dot{x}_{i}(t)=-a_{i}\left(x_{i}(t)\right)\left[b_{i}\left(x_{i}(t)\right)+f_{i}\left(t, x_{t}\right)\right], 0 \leq t \neq t_{k}, i=1,2, \ldots n, \\
& \Delta\left(x_{i}\left(t_{k}\right)\right)=I_{i k}\left(x_{i}\left(t_{k}^{-}\right)\right),
\end{aligned}
$$

where $\Delta\left(x_{i}\left(t_{k}\right)\right)=x_{i}\left(t_{k}^{+}\right)-x_{i}\left(t_{k}^{-}\right), a_{i}: \mathbb{R} \rightarrow(0, \infty), b_{i}: \mathbb{R} \rightarrow \mathbb{R}, f_{i}: \mathbb{R} \times \mathcal{D} \rightarrow \mathbb{R}$ and $I_{i k}: \mathbb{R} \rightarrow \mathbb{R}$ are continuous functions for all $k \in \mathbb{N}, 1 \leq i \leq n$, and either $\mathcal{D}=P C_{g}$ or $\mathcal{D}=B P C$. We shall also consider the non-impulsive version of $(4.1)$,

$$
\dot{x}_{i}(t)=-a_{i}\left(x_{i}(t)\right)\left[b_{i}\left(x_{i}(t)\right)+f_{i}\left(t, x_{t}\right)\right], \quad t \geq 0, i=1,2, \ldots n .
$$

For $i=1, \ldots, n$ and $k=1,2, \ldots$, we designate

$$
\widehat{I}_{i k}(u):=I_{i k}(u)+u, \quad u \in \mathbb{R} .
$$

In the sequel, we fix the maximum norm in $\mathbb{R}^{n},|x|=\max _{1 \leq i \leq n}\left|x_{i}\right|$ for $x=\left(x_{1}, \ldots, x_{n}\right) \in$ $\mathbb{R}^{n}$, and the following hypotheses will be referred to: 
(A1) there exist constants $\beta_{i}>0$ such that $\frac{b_{i}(u)-b_{i}(v)}{u-v} \geq \beta_{i}>0$, for all $u, v \in \mathbb{R}, u \neq v$, $i=1,2, \ldots, n$;

(A2) $f_{i}: \mathbb{R} \times P C_{g} \rightarrow \mathbb{R}$ are uniformly Lipschitz continuous with respect to $\varphi \in P C_{g}$, with $\left|f_{i}(t, \varphi)-f_{i}(t, \psi)\right| \leq l_{i}\|\varphi-\psi\|_{g}$, for $t \in \mathbb{R}$ and $\varphi, \psi \in P C_{g}, i=1,2, \ldots, n$;

(A3) $\beta_{i}>l_{i}, i=1,2, \ldots, n$;

(A4) $\widehat{I}_{i k}$ are Lipschitz continuous, with $\left|\widehat{I}_{i k}(u)-\widehat{I}_{i k}(v)\right| \leq \widehat{\gamma}_{i k}|u-v|$ for $u, v \in \mathbb{R}, i=$ $1, \ldots, n, k \in \mathbb{N}$, where $\widehat{I}_{i k}(u)=u+I_{i k}(u), u \in \mathbb{R}$

(A5) for $\widehat{\gamma}_{k}:=\max _{1 \leq i \leq n} \widehat{\gamma}_{i k}, \prod_{k=1}^{\infty} \max \left\{1, \widehat{\gamma}_{k}\right\}<\infty$.

For the impulsive system (4.1), the application of Theorem 3.2 yields the following existence result:

Proposition 4.1. Assume (A1)-(A4). Then the initial value problem (4.1)-(2.2) has a solution $x(t)$ defined on $[0, \infty)$.

Proof. Let $\phi \in B P C$ be given. For each $b>0$ fixed, if there is a solution $x(t)$ of (4.1)-(2.2) defined on $[0, b]$, then it follows from the proof of Lemma 4.1 that it must be uniformly bounded by a constant $M=M(b, \phi)$. The functions $a_{i}(u), b_{i}(u)$ are bounded for $u \in$ $[-M, M], 1 \leq i \leq n$, and $f_{i}(t, \phi)$ are Lipschitz continuous with respect to $\phi$, hence (3.3) is satisfied on $[0, b]$. Thus, (4.1)-(2.2) has a solution defined on $[0, b]$, for all $b>0$.

Lemma 4.2. Assume (A1), (A2) and (A3), and that for each $x \in \mathbb{R}^{n}$ the functions $t \mapsto$ $f_{i}(t, x)$ are constant on $\mathbb{R}, 1 \leq i \leq n$. Then there exists a unique equilibrium point $x^{*}=$ $\left(x_{1}^{*}, \ldots, x_{n}^{*}\right)$ of (4.2)

Proof. The result is an immediate consequence of Lemma 3.1 in [6].

Lemma 4.3. Suppose that conditions (A1)-(A5) are satisfied. Assume also that there exists a unique equilibrium point $x^{*}=\left(x_{1}^{*}, \ldots, x_{n}^{*}\right)$ of $(4.2)$, with $\widehat{I}_{i k}\left(x_{i}^{*}\right)=x_{i}^{*}$ for all $i=1, \ldots, n, k \geq 1$. Then, any solution $x(t)=x(t, 0, \phi)$ of (4.1) with initial condition $\phi \in B P C$ satisfies

$$
\left|x(t)-x^{*}\right| \leq\left\|\phi-x^{*}\right\|_{g} \prod_{k=1}^{\infty} \max \left\{1, \widehat{\gamma}_{k}\right\}, \quad t \geq 0 .
$$

Proof. Let $x^{*}=\left(x_{1}^{*}, \ldots, x_{n}^{*}\right)$ be the equilibrium of the continuous FDE (4.2). Clearly $x^{*}$ is also an equilibrium of the impulsive system (4.1) if $\widehat{I}_{i k}\left(x^{*}\right)=x^{*}$ for all $k \geq 1$ and $i=1, \ldots, n$.

Translating $x^{*}$ to the origin by the change $\tilde{x}(t)=x(t)-x^{*}$, system (4.1) becomes

$$
\begin{aligned}
& \dot{\tilde{x}}_{i}(t)=-\tilde{a}_{i}\left(\tilde{x}_{i}(t)\right)\left[\tilde{b}_{i}\left(\tilde{x}_{i}(t)\right)-\tilde{f}_{i}\left(t, \tilde{x}_{t}\right)\right], \quad 0 \leq t \neq t_{k}, i=1,2, \ldots n \\
& \Delta\left(\tilde{x}_{i}\left(t_{k}\right)\right)=\tilde{I}_{i k}\left(\tilde{x}_{i}\left(t_{k}^{-}\right)\right),
\end{aligned}
$$

where $\tilde{a}_{i}(u)=a_{i}\left(u+x_{i}^{*}\right), \tilde{b}_{i}(u)=b_{i}\left(u+x_{i}^{*}\right), \tilde{f}_{i}(t, \varphi)=f_{i}\left(t, \varphi+x^{*}\right)$ and $\tilde{I}_{i k}(u)=I_{i k}\left(u+x_{i}^{*}\right)$ with zero as the unique equilibrium, i.e., $\tilde{b}_{i}(0)+\tilde{f}_{i}(t, 0)=0$ for $i=1, \ldots n, t \in \mathbb{R}$. Clearly $b_{i}$, 
$f_{i}$ and $I_{i k}$ satisfy (A1)-(A5) if only if $\tilde{b}_{i}, \tilde{f}_{i}$ and $\tilde{I}_{i k}$ satisfy (A1)-(A5). For simplicity, instead of (4.5) we consider (4.1) subject to the conditions $b_{i}(0)+f_{i}(t, 0)=0$ and $\widehat{I}_{i k}(0)=0$ for all $t \in \mathbb{R}, i=1, \ldots, n$, and $k \in \mathbb{N}$.

Define $\widehat{I}_{k}(u)=\left(\widehat{I}_{1 k}\left(u_{1}\right), \ldots, \widehat{I}_{n k}\left(u_{n}\right)\right)$ for $u=\left(u_{1}, \ldots, u_{n}\right) \in \mathbb{R}^{n}$, where as before $\widehat{I}_{i k}(x)=$ $I_{i k}(x)+x, x \in \mathbb{R}$. We prove that hypotheses (H1) and (H2) in Lemma 4.1 are satisfied.

Consider $\psi \in P C_{g}$ with $\frac{1}{g(\theta)}|\psi(\theta)|<|\psi(0)|$ for all $\theta<0$, and let $|\psi(0)|=\psi_{i}(0)>0$ (analogous if $\left.\psi_{i}(0)<0\right)$. From (A1)-(A3), we derive

$$
\begin{aligned}
{\left[b_{i}\left(\psi_{i}(0)\right)+f_{i}(t, \psi)\right] } & =\left[b_{i}\left(\psi_{i}(0)\right)-b_{i}(0)\right]+\left[f_{i}(t, \psi)-f_{i}(t, 0)\right] \\
& \geq \beta_{i} \psi_{i}(0)-l_{i}\|\psi\|_{g}=\left(\beta_{i}-l_{i}\right)\|\psi\|_{g}>0 .
\end{aligned}
$$

Therefore, $F_{i}(t, \psi):=-a_{i}\left(\psi_{i}(0)\right)\left[b_{i}\left(\psi_{i}(0)\right)+f_{i}(t, \psi)\right]<0$, and (H1) holds. Since

$$
\left|\widehat{I}_{k}(u)\right|=\left|\widehat{I}_{k}(u)-\widehat{I}_{k}(0)\right| \leq \widehat{\gamma}_{k}|u|
$$

for $u \in \mathbb{R}^{n}$ and $\widehat{\gamma}_{k}=\max _{1 \leq i \leq n} \widehat{\gamma}_{i k}$, from (A5) we conclude that (H2) holds. From Lemma 4.1, each solution $x(t)=x(t, 0, \phi)$ with $\phi \in P C_{g}$ is bounded on $\mathbb{R}$ and satisfies (4.4).

Remark 4.2. Instead of (A2), suppose that the following condition is satisfied:

(A2') $f_{i}: \mathbb{R} \times B P C \rightarrow \mathbb{R}$ are uniformly Lipschitz continuous with respect to $\varphi \in B P C$, with $\left|f_{i}(t, \varphi)-f_{i}(t, \psi)\right| \leq l_{i}\|\varphi-\psi\|_{\infty}$, for $t \in \mathbb{R}$ and $\varphi, \psi \in B P C, i=1,2, \ldots, n$.

Then, one can verify that (4.5) satisfies (H1') instead of (H1), and from Remark 4.1 we conclude that the estimate (4.4) is replaced by $\left|x(t)-x^{*}\right| \leq\left\|\phi-x^{*}\right\|_{\infty} \prod_{k=1}^{\infty} \max \left\{1, \widehat{\gamma}_{k}\right\}, t \geq 0$.

Theorem 4.1. Assume that conditions (A1)-(A6) are satisfied, where

(A6) $\inf _{k \geq 1}\left(t_{k+1}-t_{k}\right)>0$.

Suppose also that

$$
I_{i k}\left(x_{i}^{*}\right)=0, \quad i=1, \ldots, n, k=1,2, \ldots,
$$

where $x^{*}$ is the unique equilibrium of the continuous system (4.2). Then $x^{*}$ is the unique equilibrium of the impulsive system (4.1), and is globally asymptotically stable.

Proof. From the lemma above, all solutions are bounded on $\mathbb{R}$ and the equilibrium $x^{*}$ is uniformly stable. After translating $x^{*}$ to the origin by the change $\tilde{x}(t)=x(t)-x^{*},(4.1)$ is transformed into (4.5), where for simplicity we drop the tildes and consider (4.1) subject to the conditions $b_{i}(0)+f_{i}(t, 0)=0$ and $\widehat{I}_{i k}(0)=0$ for all $t \in \mathbb{R}, i=1, \ldots, n$, and $k \in \mathbb{N}$.

It remains to prove that $x=0$ is globally attractive. Let $x(t)=x(t, 0, \varphi)$ be the solution of (4.1) with an initial condition $x_{0}=\varphi \in B P C, x(t)=\left(x_{1}(t), \ldots, x_{n}(t)\right)$ for $t \in \mathbb{R}$.

Define $-v_{i}=\liminf _{t \rightarrow \infty} x_{i}(t)$ and $u_{i}=\limsup _{t \rightarrow \infty} x_{i}(t)$ for $i=1, \ldots, n$, and $v=$ $\max _{i}\left\{v_{i}\right\}$ and $u=\max _{i}\left\{u_{i}\right\}$. Note that $-\infty<-v \leq u<\infty$. Suppose that $|v| \leq u$, then $\max (u, v)=u$ (the case $|u| \leq v$ is analogous). It is sufficient to prove that $u=0$, which is done in several steps.

Claim 1. For each $\varepsilon>0$, there exists $T>0$ such that $\left\|x_{t}\right\|_{g} \leq u+\varepsilon$ for all $t \geq T$.

Since $x(t)$ is uniformly bounded, take $M>0$ such that $|x(t)| \leq M$ for all $t \in \mathbb{R}$. Now, fix any $\varepsilon>0$. There is $T_{0}>0$ such that $|x(t)| \leq u+\varepsilon$ for all $t \geq T_{0}$. Let $T_{1}>T_{0}$ be sufficiently large so that $M / g\left(-T_{1}\right)<u+\varepsilon$, and take $T=2 T_{1}$. 
Consider $t \geq T$. If $s \leq-T_{1}$, then

$$
\frac{|x(t+s)|}{g(s)} \leq \frac{M}{g\left(-T_{1}\right)}<u+\varepsilon ;
$$

if $-T_{1} \leq s \leq 0$, then $t+s \geq T_{1}$ and therefore

$$
\frac{|x(t+s)|}{g(s)} \leq|x(t+s)| \leq u+\varepsilon .
$$

From (4.7) and (4.8), we obtain $\left\|x_{t}\right\|_{g} \leq u+\varepsilon$ for $t \geq T$. This proves Claim 1.

Next, since $a_{i}(x)>0$ for all $x \in \mathbb{R}$ and $x_{i}(t)$ is bounded, we observe that there is $m>0$ such that $a_{i}\left(x_{i}(t)\right) \geq m$ for $t \geq 0$ and $i=1, \ldots, n$. If $u>0$, then $\eta>0$, where

$$
\eta:=m\left(\beta_{i}-l_{i}\right) u / 2 .
$$

Claim 2. If $u>0$ and $s_{j} \rightarrow \infty, x_{i}\left(s_{j}\right) \rightarrow u$ (respectively $x_{i}\left(s_{j}\right) \rightarrow-u$ ) as $j \rightarrow \infty$ for some $i=1, \ldots, n$, with $s_{j} \neq t_{k}$ for all $j, k \in \mathbb{N}$, then there exists $j_{1} \in \mathbb{N}$ such that $\dot{x}_{i}\left(s_{j}\right) \leq-\eta$ (respectively $\dot{x}_{i}\left(s_{j}\right) \geq \eta$ ) for all $j \geq j_{1}$.

Suppose that $u>0$ and that $x_{i}\left(s_{j}\right) \rightarrow u$ for some sequence $\left(s_{j}\right)$ with $s_{j} \rightarrow \infty$. Using (A1), (A2) and (A3) and for $\varepsilon \in\left(0, \frac{\left(\beta_{i}-l_{i}\right) u}{2\left(\beta_{i}+l_{i}\right)}\right)$, from Claim 1 we have

$$
\begin{aligned}
b_{i}\left(x_{i}\left(s_{j}\right)\right)+f_{i}\left(s_{j}, x_{s_{j}}\right) & =\left(b_{i}\left(x_{i}\left(s_{j}\right)\right)-b_{i}(0)\right)+\left(f_{i}\left(s_{j}, x_{s_{j}}\right)-f_{i}\left(s_{j}, 0\right)\right) \\
& \geq \beta_{i} x_{i}\left(s_{j}\right)-l_{i}\left\|x_{s_{j}}\right\|_{g} \geq \beta_{i}(u-\varepsilon)-l_{i}(u+\varepsilon) \\
& =\left(\beta_{i}-l_{i}\right) u-\left(\beta_{i}+l_{i}\right) \varepsilon \geq\left(\beta_{i}-l_{i}\right) u / 2>0
\end{aligned}
$$

for $j$ sufficiently large. Thus,

$$
\dot{x}_{i}\left(s_{j}\right) \leq-a_{i}\left(x_{i}\left(s_{j}\right)\right)\left(\beta_{i}-l_{i}\right) u / 2 \leq-m\left(\beta_{i}-l_{i}\right) u / 2=-\eta
$$

for $j$ sufficiently large. If $x\left(s_{j}\right) \rightarrow-u$, in a similar way we conclude that $\dot{x}_{i}\left(s_{j}\right) \geq \eta$, and claim 2 is proven.

Now fix $i \in\{1, \ldots, n\}$ such that $u_{i}=u$. Then, there is a sequence $\left(s_{j}\right)_{j}$ such that $s_{j} \nearrow \infty$ and

$$
\max \left\{x_{i}\left(s_{j}\right), x_{i}\left(s_{j}^{+}\right)\right\} \rightarrow u \quad \text { as } \quad j \rightarrow \infty .
$$

Since $x_{i}(t)$ is continuous on each interval $\left(t_{k-1}, t_{k}\right]$, we may assume that $x_{i}\left(s_{j}\right) \rightarrow u$ as $j \rightarrow \infty$ with

$$
s_{j} \in\left(t_{k(j)-1}, t_{k(j)}\right) .
$$

for some subsequence $\left(t_{k(j)}\right)_{j}$ of $\left(t_{k}\right)_{k}$.

Claim 3. If $u>0$, then $\lim \sup _{j \rightarrow \infty} \max \left\{x_{i}\left(t_{k(j)}\right), \mid x_{i}\left(t_{k(j)-1} \mid\right)\right\}=u$.

We first prove that $\limsup _{j \rightarrow \infty} \max \left\{x_{i}\left(t_{k(j)}\right), x_{i}\left(t_{k(j)-1}^{+}\right)\right\}=u$. Otherwise, consider a subsequence of $s_{j}$, still denoted by $s_{j}$, such that $\max \left(\left\{x_{i}(t): t \in\left(t_{k(j)-1}, t_{k(j)}\right]\right\} \cup\right.$ $\left.\left\{x\left(t_{k(j)-1}^{+}\right)\right\}\right)=x_{i}\left(\bar{s}_{j}\right)$, for some $\bar{s}_{j} \in\left(t_{k(j)-1}, t_{k(j)}\right)$. So we have $x_{i}\left(\bar{s}_{j}\right) \geq x_{i}\left(s_{j}\right) \rightarrow u$ as 
$j \rightarrow \infty$ and $\dot{x}_{i}\left(\bar{s}_{j}\right)=0$. Clearly, we can replace $s_{j}$ with $\bar{s}_{j}$ in (4.9), which leads to a contradiction.

Thus $\limsup _{j \rightarrow \infty} \max \left\{x_{i}\left(t_{k(j)}\right), x_{i}\left(t_{k(j)-1}^{+}\right)\right\}=u$. On the other hand, from (A5) it is known that $\log \left(\max \left\{1, \widehat{\gamma}_{k}\right\}\right) \rightarrow 0$ as $k \rightarrow \infty$, hence $\lim \sup \widehat{\gamma}_{k} \leq 1$. For any $\varepsilon>0$ small, we get

$$
\left|x_{i}\left(t_{k(j)-1}^{+}\right)\right| \leq \widehat{\gamma}_{k(j)-1}\left|x_{i}\left(t_{k(j)-1}\right)\right| \leq u+\varepsilon
$$

for $j$ large, we conclude that $\lim \sup _{j \rightarrow \infty} \max \left\{x_{i}\left(t_{k(j)}\right), \mid x_{i}\left(t_{k(j)-1} \mid\right)\right\}=u$.

We finally prove:

Claim 4. $u=0$.

By way of contradiction, suppose again that $u>0$.

Claim 2 and the above arguments also show that, if a subsequence $x_{i}\left(t_{k(j)}\right)$ of $x_{i}\left(t_{k}\right)$ converges to $u$ (respectively $-u$ ), then, for $j$ large, there are no local maximum (respectively minimum) points of $x_{i}(t)$ in the intervals $\left(t_{k(j)-1}, t_{k(j)}\right)$.

For some subsequence of $t_{k(j)}$, still designated by $t_{k(j)}$, from Claim 3 , we obtain one of the following cases: (i) $x_{i}\left(t_{k(j)}\right) \rightarrow u$; (ii) $x_{i}\left(t_{k(j)-1}\right) \rightarrow u$; or (iii) $x_{i}\left(t_{k(j)-1}\right) \rightarrow-u$. We consider separately these three situations.

In Case $(\mathrm{i}), x_{i}(t)$ is strictly decreasing on $\left(t_{k(j)-1}, t_{k(j)}\right]$. Now, define the functions

$$
\varphi_{j}:[0, \sigma] \rightarrow \mathbb{R}, \varphi_{j}(s)=x_{i}\left(t_{k(j)}-\sigma+s\right),
$$

where by (A6) we choose $\sigma$ such that $0<\sigma<\inf _{k \geq 1}\left(t_{k+1}-t_{k}\right)$. Thus, $\varphi_{j} \in C^{1}([0, \sigma], \mathbb{R})$, $\varphi_{j}(\sigma) \rightarrow u$, and $\varphi_{j}(s)$ decreasing in $[0, \sigma]$, for each $j$. Thus $\varphi_{j}(s) \rightarrow u$ for each $s \in[0, \sigma]$. As in the proof of Claim 2, for $j$ sufficiently large and $t \in\left(t_{k(j)-1}, t_{k(j)}\right)$ we get

$$
b_{i}\left(x_{i}(t)\right)+f_{i}\left(t, x_{t}\right) \geq \beta_{i} x_{i}(t)-l_{i}\left\|x_{t}\right\|_{g} \geq \beta_{i} x_{i}\left(t_{k(j)}\right)-l_{i}\left\|x_{t}\right\|_{g} \geq\left(\beta_{i}-l_{i}\right) u / 2,
$$

hence $\dot{\varphi}_{j}(s) \leq-\eta<0$ for all $s \in[0, \sigma)$. Clearly, this is not possible, since it leads to $\varphi_{j}(0) \geq \varphi_{j}(\sigma)+\eta \sigma \rightarrow u+\eta \sigma>u$, a contradiction.

Case (ii) is treated as Case (i), by replacing the intervals $\left(t_{k(j)-1}, t_{k(j)}\right]$ with $\left(t_{k(j)-2}, t_{k(j)-1}\right]$.

In Case (iii), we derive that $x_{i}(t)$ is strictly increasing on $\left(t_{k(j)-2}, t_{k(j)-1}\right]$. We proceed as in Case (i), with $\left(t_{k(j)-2}, t_{k(j)-1}\right]$ instead of $\left(t_{k(j)-1}, t_{k(j)}\right]$, and where now we obtain $\varphi_{j}(s) \rightarrow-u, \dot{\varphi}_{j}(s) \geq \eta>0$ for all $s \in[0, \sigma)$, yielding a contradiction in a similar way.

Remark 4.3. In Claim 2, in fact one can consider $s_{j}=t_{k}$ for some $k, j \in \mathbb{N}$, in which case $\dot{x}_{i}\left(s_{j}\right)$ means the left derivative of $x_{i}(t)$ at $t=t_{k}$. For $f$ regular enough, note that a solution $x(t)$ of (4.1) necessarily has left and right derivatives at the points $t=t_{k}$, since the function $y(t)$ with components $y_{i}(t)=x_{i}(t)-\sum_{k: 0<t_{k}<t} I_{i k}\left(x_{i}\left(t_{k}\right)\right)$ is absolutely continuous on each interval $\left[t_{k-1}, t_{k}\right]$.

Remark 4.4. If the functions $I_{i k}: \mathbb{R} \rightarrow \mathbb{R}$ are Lispchitz continuous,

(A4') $\left|I_{i k}(u)-I_{i k}(v)\right| \leq \gamma_{i k}|u-v|$ for $u, v \in \mathbb{R}, i=1, \ldots, n, k \in \mathbb{N}$,

then (A4) holds with $\widehat{\gamma}_{i k} \leq\left(1+\gamma_{i k}\right)$. If in addition

(A5') $\sum_{k} \gamma_{k}<\infty$ for $\gamma_{k}:=\max _{1 \leq i \leq n} \gamma_{i k}$,

then the series $\sum_{k} \log \left(1+\gamma_{k}\right)$ is also convergent, and consequently (A5) is satisfied. Therefore, Theorem 4.1 is still valid if we replace assumptions (A4), (A5) by (A4'), (A5'). 
We shall now study the global exponential stability of an equilibrium point of the impulsive system (4.1). For this purpose, in the remaining part of this section we shall consider the phase space $P C_{g}$, with

$$
g(s)=e^{-\alpha s}, \quad s \in(-\infty, 0],
$$

for some $\alpha>0$, and denote $P C_{g}$ by $P C_{\alpha}$ and $\|\cdot\|_{g}$ by $\|\cdot\|_{\alpha}$

Lemma 4.4. Assume (A1), (A2), (A3), and

(A7) $a_{i}(u) \geq \underline{a_{i}}>0$, for all $u \in \mathbb{R}, i=1,2, \ldots, n$.

Suppose also that $x^{*}$ is the unique equilibrium point of (4.2). Consider a solution $x$ : $(-\infty, b] \rightarrow \mathbb{R}^{n}$ of the non-impulsive system (4.2) on $[a, b]$ with $x_{a} \in P C_{\alpha}(-\infty<a<b \leq \infty)$. If there exist constants $q>0$ and $\varepsilon \in(0, \alpha]$ with $\varepsilon<\min _{i}\left\{\underline{a_{i}}\left(\beta_{i}-l_{i}\right)\right\}$, such that

$$
\left|x(t)-x^{*}\right| \leq q e^{-\varepsilon(t-a)} \quad \text { for } \quad t \in(-\infty, a]
$$

then

$$
\left|x(t)-x^{*}\right| \leq q e^{-\varepsilon(t-a)} \quad \text { for } \quad t \in(-\infty, b] .
$$

Proof. After the change $y(t)=x(t)-x^{*}$, we may assume that the equilibrium point is zero, i.e., we consider (4.2) subject to the constraints $b_{i}(0)+f_{i}(t, 0)=0, t \in \mathbb{R}, 1 \leq i \leq n$.

By contradiction, suppose that (4.12) does not hold. Consequently there are $\delta>0$, $m \in\{1, \ldots, n\}$, and $t^{*} \in(a, b]$ such that

$$
\left|y_{m}\left(t^{*}\right)\right|=(q+\delta) e^{-\varepsilon\left(t^{*}-a\right)} \text { and }\left|y_{i}(t)\right|<(q+\delta) e^{-\varepsilon(t-a)}, \quad t<t^{*}, i=1, \ldots, n .
$$

Assume that $y_{m}\left(t^{*}\right)>0$ (the situation $y_{m}\left(t^{*}\right)<0$ is analogous). Denoting $z(t):=(q+$ $\delta) e^{-\varepsilon(t-a)}, t \in[a, b]$, we have

$$
y_{m}^{\prime}\left(t^{*}\right) \geq z^{\prime}\left(t^{*}\right) .
$$

On the other hand, using the hypotheses (A1)-(A3) and (A7), we have

$$
\begin{aligned}
y_{m}^{\prime}\left(t^{*}\right) & =-a_{m}\left(y_{m}\left(t^{*}\right)\right)\left[b_{m}\left(y_{m}\left(t^{*}\right)\right)+f_{m}\left(t^{*}, y_{t^{*}}\right)\right] \\
& =-a_{m}\left(y_{m}\left(t^{*}\right)\right)\left[b_{m}\left(y_{m}\left(t^{*}\right)\right)-b_{m}(0)+f_{m}\left(t^{*}, y_{t^{*}}\right)-f_{m}\left(t^{*}, 0\right)\right] \\
& \leq-\underline{a_{m}}\left[\beta_{m} y_{m}\left(t^{*}\right)-l_{m}\left\|y_{t^{*}}\right\|_{\alpha}\right] \\
& \leq-\underline{a_{m}}\left[\beta_{m} z\left(t^{*}\right)-l_{m} \sup _{s \leq 0} \frac{(q+\delta) e^{-\varepsilon\left(t^{*}+s-a\right)}}{e^{-\alpha s}}\right] \\
& \leq-\underline{a_{m}}\left[\beta_{m} z\left(t^{*}\right)-l_{m}(q+\delta) e^{-\varepsilon\left(t^{*}-a\right)}\right] \\
& =-\underline{a_{m}}\left(\beta_{m}-l_{m}\right) z\left(t^{*}\right)<-\varepsilon z\left(t^{*}\right)=z^{\prime}\left(t^{*}\right),
\end{aligned}
$$

which is a contradiction.

Theorem 4.2. Assume that there is an equilibrium $x^{*}$ of (4.1). Assume also (A1)-(A4), (A7) and

(A8) for $\widehat{\gamma}_{k}:=\max _{1 \leq i \leq n} \widehat{\gamma}_{i k}$ and $\widehat{\gamma}_{i k}$ as in (A4) and some $k_{0} \in \mathbb{N}$,

$$
\eta:=\sup _{k \geq k_{0}}\left(\frac{\log \left(\max \left\{1, \widehat{\gamma}_{k}\right\}\right)}{t_{k}-t_{k-1}}\right)<\min _{i}\left\{\underline{a_{i}}\left(\beta_{i}-l_{i}\right)\right\},
$$

where the space $P C_{g}$ in (A2) is $P C_{g}=P C_{\varepsilon}$ for some $\varepsilon>\eta$. Then the equilibrium $x^{*}$ of (4.1) is globally exponentially stable. 
Proof. For $\eta$ as in (4.13), choose $\alpha \in(0, \varepsilon]$ such that

$$
\eta<\alpha<\min _{i}\left\{\underline{a_{i}}\left(\beta_{i}-l_{i}\right)\right\} .
$$

Clearly, (A2) still holds with $P C_{\varepsilon}$ replaced by $P C_{\alpha}$, with the same Lipschitz constants $l_{i}$. Write $\eta_{k}=\max \left\{1, \widehat{\gamma}_{k}\right\}, k=1,2, \ldots$ As above, we may assume that $x^{*}=0$ is the unique equilibrium point of (4.1). Let $y(t)$ be a solution of (4.1) defined on $\mathbb{R}$, with $y_{0} \in P C_{\alpha}$. Then,

$$
|y(t)|_{\infty} \leq\left\|y_{0}\right\|_{\alpha} e^{-\alpha t}, \quad \text { for } \quad t \in(-\infty, 0] .
$$

From Lemma 4.4, we conclude that

$$
|y(t)|_{\infty} \leq\left\|y_{0}\right\|_{\alpha} e^{-\alpha t} \quad \text { for } \quad t \in\left(-\infty, t_{1}\right] .
$$

Next, we observe that $\left|y\left(t_{1}^{+}\right)\right|_{\infty}=\left|y_{i}\left(t_{1}^{+}\right)\right|$, for some $i=1, \ldots, n$, and

Consequently,

$$
\begin{aligned}
\left|y\left(t_{1}^{+}\right)\right|_{\infty} & =\left|y_{i}\left(t_{1}^{+}\right)\right|=\left|I_{i 1}\left(y_{i}\left(t_{1}\right)\right)+y_{i}\left(t_{1}\right)\right|=\left|\widehat{I}_{i 1}\left(y_{i}\left(t_{1}\right)\right)\right| \\
& \leq \widehat{\gamma}_{i 1}\left|y_{i}\left(t_{1}\right)\right| \leq \widehat{\gamma}_{i 1}\left\|y_{0}\right\|_{\alpha} e^{-\alpha t_{1}} \leq \eta_{1}\left\|y_{0}\right\|_{\alpha} e^{-\alpha t_{1}} .
\end{aligned}
$$

$$
|y(t)|_{\infty} \leq \eta_{1}\left\|y_{0}\right\|_{\alpha} e^{-\alpha t_{1}} e^{-\alpha\left(t-t_{1}\right)}, \quad t \in\left(-\infty, t_{1}^{+}\right]
$$

and, again from Lemma 4.4, we conclude that

$$
|y(t)|_{\infty} \leq \eta_{1}\left\|y_{0}\right\|_{\alpha} e^{-\alpha t} \quad \text { for } \quad t \in\left(-\infty, t_{2}\right] .
$$

Noting that $\left|y\left(t_{2}^{+}\right)\right|_{\infty}=\left|y_{i}\left(t_{2}^{+}\right)\right|$, for some $i=1, \ldots, n$, then

$$
\left|y\left(t_{2}^{+}\right)\right|_{\infty}=\left|\widehat{I}_{i 2}\left(y_{i}\left(t_{2}\right)\right)\right| \leq \widehat{\gamma}_{i 2}\left|y_{i}\left(t_{2}\right)\right| \leq \eta_{2} \eta_{1}\left\|y_{0}\right\|_{\alpha} e^{-\alpha t_{2}} .
$$

Iterating the above procedure, we obtain

$$
|y(t)|_{\infty} \leq \eta_{1} \eta_{2} \cdots \eta_{k-1}\left\|y_{0}\right\|_{\alpha} e^{-\alpha t}, t \in\left(t_{k-1}, t_{k}\right], k=1,2, \ldots,
$$

where $t_{0}=0$ and $\eta_{0}=1$. The definition of $\eta$ in (4.13) yields $\log \eta_{k} \leq \eta\left(t_{k}-t_{k-1}\right)$, hence

$$
\eta_{k} \leq e^{\eta\left(t_{k}-t_{k-1}\right)}, \quad k \geq k_{0} .
$$

Thus, for $t \in\left(t_{k-1}, t_{k}\right]$ and $k>k_{0}$,

$$
|y(t)|_{\infty} \leq \eta_{1} \cdots \eta_{k_{0}-1}\left\|y_{0}\right\|_{\alpha} e^{\eta t_{k-1}} e^{-\alpha t} \leq \eta_{1} \cdots \eta_{k_{0}-1}\left\|y_{0}\right\|_{\alpha} e^{-(\alpha-\eta) t},
$$

and, consequently

$$
|y(t)|_{\infty} \leq \eta_{1} \cdots \eta_{k_{0}-1}\left\|y_{0}\right\|_{\alpha} e^{-(\alpha-\eta) t}, \quad t \geq 0
$$

Corollary 4.1. In the space $P C_{\varepsilon}$ for some $\varepsilon>0$, assume that there is an equilibrium point $x^{*}$ of (4.1) and that (A1)-(A7) hold. Then $x^{*}$ is globally exponentially stable.

Proof. Observe that (A5) and (A6) imply (A8).

Remark 4.5. Consider system (4.1) in some space $P C_{\varepsilon}$ with $\varepsilon>0$, and suppose that the functions $a_{i}(x)$ are bounded from below by some positive constant (i.e., (A7) holds). Then, the above corollary shows that the sufficient conditions in Theorem 4.1 for the global asymptotic stability of an equlibrium $x^{*}$ are stronger then the requirements in Theorem 4.2 for its global exponential stability, which seems to indicate that Theorem 4.1 does not provide a good criterion. However, in the applications given in the next section it will be apparent that it is much more restrictive to set (4.1) in the framework of a space $P C_{\varepsilon}=P C_{g}$ with $g(s)=e^{-\varepsilon s}(\varepsilon>0)$, then in a generic space $P C_{g}$ with $g$ satisfying (g1)-(g3). 


\section{Applications}

Here, we apply the previous results to the following impulsive generalized Cohen-Grossberg neural network model with both time-varing delays and distributed delays:

$$
\begin{gathered}
\dot{x}_{i}(t)=-a_{i}\left(x_{i}(t)\right)\left[b_{i}\left(x_{i}(t)\right)+\sum_{j=1}^{n} \sum_{p=1}^{P}\left(h_{i j}^{(p)}\left(x_{j}\left(t-\tau_{i j}^{(p)}(t)\right)\right)+\right.\right. \\
\left.\left.\quad+f_{i j}^{(p)}\left(\int_{-\infty}^{0} g_{i j}^{(p)}\left(x_{j}(t+s)\right) d \eta_{i j}^{(p)}(s)\right)\right)\right], 0 \leq t \neq t_{k}, \\
\Delta\left(x_{i}\left(t_{k}\right)\right)=I_{i k}\left(x_{i}\left(t_{k}^{-}\right)\right), i=1, \ldots n, k \in \mathbb{N},
\end{gathered}
$$

where $t_{k} \nearrow \infty$ as $k \rightarrow \infty, a_{i}: \mathbb{R} \rightarrow(0, \infty), b_{i}, h_{i j}^{(p)}, f_{i j}^{(p)}, g_{i j}^{(p)}, I_{i k}: \mathbb{R} \rightarrow \mathbb{R}, \tau_{i j}^{(p)}:[0, \infty) \rightarrow$ $[0, \infty)$ are continuous functions with $\tau_{i j}^{(p)}(t) \leq \tau_{i j}^{(p)} \leq \tau$ for some $\tau>0$, and $\eta_{i j}^{(p)}:(-\infty, 0] \rightarrow$ $\mathbb{R}$ are non-decreasing bounded functions, normalized so that $\eta_{i j}^{(p)}(0)-\eta_{i j}^{(p)}(-\infty)=1$, for all $i, j \in\{1, \ldots, n\}, p \in\{1, \ldots, P\}$. For (5.1), in the sequel we further assume that the functions $b_{i}$ satisfy (A1), $I_{i k}$ satisfy (A4), and that $h_{i j}^{(p)}, f_{i j}^{(p)}, g_{i j}^{(p)}$ are Lipschitzian with Lipschitz constants $\zeta_{i j}^{(p)}, \mu_{i j}^{(p)}, \sigma_{i j}^{(p)}$, respectively.

Model (5.1) was introduced in [28] and is particulary relevant in terms of applications, since it includes different types of neural network models as subclasses, as we shall illustrate with several examples.

We define the square real matrices,

$$
B=\operatorname{diag}\left(\beta_{1}, \ldots, \beta_{n}\right), \quad L=\left[l_{i j}\right] \text { and } N=B-L,
$$

where $\beta_{1}, \ldots, \beta_{n}$ are as in $(\mathrm{A} 1)$ and $l_{i j}=\sum_{p=1}^{P} \zeta_{i j}^{(p)}+\mu_{i j}^{(p)} \sigma_{i j}^{(p)}$.

Proposition 5.1. Consider (5.1) under the hypotheses assumed above. If $N$ is a nonsingular M-matrix, then the model (5.1) has a unique equilibrium point $x^{*}=\left(x_{1}^{*}, \ldots, x_{n}^{*}\right)$.

Proof. See [28].

In addition, in what follows we always assume that if $x^{*}$ is an equilibrium point of (5.1), then it satisfies

$$
I_{i k}\left(x_{i}^{*}\right)=0, \quad i=1, \ldots, n, k \in \mathbb{N} .
$$

This means that $x^{*}$ is also an equilibrium point of the impulsive model (5.1)-(5.2).

Theorem 5.1. Consider (5.1)-(5.2) under the hypotheses assumed above. In addition, assume the hypotheses (A5), (A6), and that the matrix $N$, defined in (5.3), is a non-singular M-matrix.

Then there is a unique equilibrium point $x^{*} \in \mathbb{R}^{n}$ of (5.1)-(5.2) which is globally asymptotically stable.

Proof. The existence and uniqueness of the equilibrium point of (5.1)-(5.2) comes from Proposition 5.1 and (5.4). 
As $N$ is a non-singular M-matrix, then (see [7]) there is $d=\left(d_{1}, \ldots, d_{n}\right)>0$ such that $N d>0$, i.e.,

$$
\beta_{i} d_{i}>\sum_{j=1}^{n} l_{i j} d_{j}, \quad i=1, \ldots, n,
$$

hence there is $\delta>0$ such that

$$
\beta_{i} d_{i}>\sum_{j=1}^{n} d_{j} \sum_{p=1}^{P} \zeta_{i j}^{(p)}+\mu_{i j}^{(p)} \sigma_{i j}^{(p)}(1+\delta), \quad i=1, \ldots, n .
$$

Since $\int_{-\infty}^{0} d \eta_{i j}^{(p)}(s)<1+\delta$ for $i, j=1, \ldots, n, p=1, \ldots, P$, from Lemma 4.1 in [6] we conclude that there is $g:(-\infty, 0] \rightarrow[1, \infty)$ satisfying (g1)-(g3) such that

$$
\int_{-\infty}^{0} g(s) d \eta_{i j}^{(p)}(s)<1+\delta \quad \text { and } \quad g(-\tau)=1
$$

The change $y_{i}(t)=d_{i}^{-1} x_{i}(t)$ transforms (5.1)-(5.2) into the system

$$
\begin{gathered}
\dot{y}_{i}(t)=-a_{i}\left(d_{i} y_{i}(t)\right) d_{i}^{-1}\left[b_{i}\left(d_{i} y_{i}(t)\right)+\sum_{j=1}^{n} \sum_{p=1}^{P}\left(h_{i j}^{(p)}\left(d_{j} y_{j}\left(t-\tau_{i j}^{(p)}(t)\right)\right)+\right.\right. \\
\left.\left.+f_{i j}^{(p)}\left(\int_{-\infty}^{0} g_{i j}^{(p)}\left(d_{j} y_{j}(t+s)\right) d \eta_{i j}^{(p)}(s)\right)\right)\right], 0 \leq t \neq t_{k}, \\
\Delta\left(y_{i}\left(t_{k}\right)\right)=d_{i}^{-1} I_{i k}\left(d_{i} y_{i}\left(t_{k}^{-}\right)\right), \quad i=1, \ldots n, k \in \mathbb{N}
\end{gathered}
$$

for which we consider $P C_{g}$ as the phase space. For each $i \in\{1, \ldots, n\}$, define

$$
\begin{gathered}
\bar{f}_{i}(t, \phi)=d_{i}^{-1} \sum_{j=1}^{n} \sum_{p=1}^{P}\left(h_{i j}^{(p)}\left(d_{j} \phi_{j}\left(-\tau_{i j}^{(p)}(t)\right)\right)+f_{i j}^{(p)}\left(\int_{-\infty}^{0} g_{i j}^{(p)}\left(d_{j} \phi_{j}(s)\right) d \eta_{i j}^{(p)}(s)\right)\right), \\
\bar{a}_{i}(u)=a_{i}\left(d_{i} u\right), \quad \bar{b}_{i}(u)=d_{i}^{-1} b_{i}\left(d_{i} u\right), \quad \bar{I}_{i k}(u)=d_{i}^{-1} I_{i k}\left(d_{i} u\right)
\end{gathered}
$$

for $\phi \in P C_{g}, t \geq 0$, and $u \in \mathbb{R}$. System (5.6)-(5.7) is written as

$$
\begin{aligned}
& \dot{y}_{i}(t)=-\bar{a}_{i}\left(y_{i}(t)\right)\left[\bar{b}_{i}\left(y_{i}(t)\right)+\bar{f}_{i}\left(t, y_{t}\right)\right], \quad 0 \leq t \neq t_{k}, \\
& \Delta\left(y_{i}\left(t_{k}\right)\right)=\bar{I}_{i k}\left(y_{i}\left(t_{k}^{-}\right)\right), \quad i=1, \ldots n, k \in \mathbb{N}
\end{aligned}
$$

which has the form (4.1). Clearly $b_{i}$ and $I_{i k}$ satisfy (A1), (A4), and (A5) if and only if $\bar{b}_{i}$ and $\bar{I}_{i k}$ satisfy (A1), (A4), and (A5) with the same constants $\beta_{i}$ and $\hat{\gamma}_{i k}$.

For $\varphi, \phi \in P C_{g}$ and $t \geq 0$, since $h_{i j}^{(p)}, f_{i j}^{(p)}, g_{i j}^{(p)}$ are Lipschitz functions and $\eta_{i j}^{(p)}$ are 
non-decreasing, we have

$$
\begin{aligned}
& \begin{array}{l}
\left|\bar{f}_{i}(t, \phi)-\bar{f}_{i}(t, \varphi)\right| \leq d_{i}^{-1} \sum_{j=1}^{n} \sum_{p=1}^{P}\left(\zeta_{i j}^{(p)} d_{j}\left|\phi_{j}\left(-\tau_{i j}^{(p)}(t)\right)-\varphi_{j}\left(-\tau_{i j}^{(p)}(t)\right)\right|\right. \\
\left.\quad+\mu_{i j}^{(p)} \int_{-\infty}^{0}\left|g_{i j}^{(p)}\left(d_{j} \phi_{j}(s)\right)-g_{i j}^{(p)}\left(d_{j} \varphi_{j}(s)\right)\right| d \eta_{i j}^{(p)}(s)\right) \\
\leq d_{i}^{-1} \sum_{j=1}^{n} \sum_{p=1}^{P}\left(\zeta_{i j}^{(p)} d_{j} \frac{\left|\left(\phi_{j}-\varphi_{j}\right)\left(-\tau_{i j}^{(p)}(t)\right)\right|}{g\left(-\tau_{i j}^{(p)}(t)\right)} g(-\tau)\right. \\
\left.\quad+d_{j} \mu_{i j}^{(p)} \sigma_{i j}^{(p)} \int_{-\infty}^{0} g(s) \frac{\left|\left(\phi_{j}-\varphi_{j}\right)(s)\right|}{g(s)} d \eta_{i j}^{(p)}(s)\right) \\
\leq\left[d_{i}^{-1} \sum_{j=1}^{n} d_{j} \sum_{p=1}^{P}\left(\zeta_{i j}^{(p)}+\mu_{i j}^{(p)} \sigma_{i j}^{(p)} \int_{-\infty}^{0} g(s) d \eta_{i j}^{(p)}(s)\right)\right]\|\phi-\varphi\|_{g} .
\end{array}
\end{aligned}
$$

This means that

$$
\left|\bar{f}_{i}(t, \phi)-\bar{f}_{i}(t, \varphi)\right| \leq l_{i}\|\phi-\varphi\|_{g}, \quad i=1, \ldots, n,
$$

with $l_{i}:=d_{i}^{-1} \sum_{j=1}^{n} d_{j} \sum_{p=1}^{P}\left(\zeta_{i j}^{(p)}+\mu_{i j}^{(p)} \sigma_{i j}^{(p)}(1+\delta)\right)$, then each $\bar{f}_{i}$ satisfies (A2). On the other hand, (5.5) implies that (A3) holds. The conclusion follows now from Theorem 4.1.

Theorem 5.2. Consider (5.1)-(5.2) under the hypotheses above. In addition, suppose that:

(i) the functions $a_{i}$ satisfy (A7);

(ii) there is $k^{*} \in \mathbb{N}$ such that the conditions

$$
\int_{-\infty}^{0} e^{-\gamma s} d \eta_{i j}^{(p)}(s)<\infty, \quad i, j=1, \ldots, n, p=1, \ldots, P
$$

hold for some $\gamma>\eta:=\sup _{k \geq k^{*}}\left(\frac{\log \left(\max \left\{1, \widehat{\gamma}_{k}\right\}\right)}{t_{k}-t_{k-1}}\right)$, where $\widehat{\gamma}_{k}:=\max _{1 \leq i \leq n} \widehat{\gamma}_{i k}$;

(iii) the matrix

$$
M=\operatorname{diag}\left(\beta_{1}-\frac{\eta}{\underline{a_{1}}}, \ldots, \beta_{n}-\frac{\eta}{\underline{a_{n}}}\right)-\left[n_{i j}\right]
$$

where $n_{i j}=\sum_{p=1}^{P}\left(\zeta_{i j}^{(p)} e^{\eta \tau_{i j}^{(p)}}+\mu_{i j}^{(p)} \sigma_{i j}^{(p)} \int_{-\infty}^{0} e^{-\eta s} d \eta_{i j}^{(p)}(s)\right)$, is a non-singular M-matrix.

Then, there is a unique equilibrium point $x^{*}$ of (5.1)-(5.2) which is globally exponentially stable.

Proof. Let $N$ be defined as in (5.3). As $N \geq M$ and $M$ is a non-singular M-matrix, then $N$ is also a non-singular M-matrix. From Proposition 5.1 and assuming (5.4), we conclude that the impulsive system (5.1)-(5.2) has a unique equilibrium point $x^{*} \in \mathbb{R}^{n}$. 
Since $M$ is a non-singular M-matrix, there is $d=\left(d_{1}, \ldots, d_{n}\right)>0$ such that (see [7])

$$
\left(\beta_{i}-\frac{\eta}{\underline{a_{i}}}\right) d_{i}-\sum_{j=1}^{n} d_{j} \sum_{p=1}^{P}\left(\zeta_{i j}^{(p)} e^{\eta \tau_{i j}^{(p)}}+\mu_{i j}^{(p)} \sigma_{i j}^{(p)} \int_{-\infty}^{0} e^{-\eta s} d \eta_{i j}^{(p)}(s)\right)>0,
$$

for all $i=1, \ldots, n$, which is equivalent to

$$
C_{i}(\eta):=\eta-\underline{a_{i}}\left(\beta_{i}-d_{i}^{-1} \sum_{j=1}^{n} d_{j} \sum_{p=1}^{P}\left(\zeta_{i j}^{(p)} e^{\eta \tau_{i j}^{(p)}}+\mu_{i j}^{(p)} \sigma_{i j}^{(p)} \int_{-\infty}^{0} e^{-\eta s} d \eta_{i j}^{(p)}(s)\right)\right)<0 .
$$

As the function $F_{i j}^{(p)}(t):=\int_{-\infty}^{0} e^{-t s} d \eta_{i j}^{(p)}(s)$ is continuous (see [6], proof of Theorem 4.3) and non-decreasing on $[\eta, \gamma]$, we conclude that each $C_{i}$ is also continuous and non-decreasing on $[\eta, \gamma]$ and therefore there is $\varepsilon>\eta$ such that

$$
C_{i}(\varepsilon)<0, \quad i=1, \ldots, n .
$$

As in the proof above, the change $y_{i}(t)=d_{i}^{-1} x_{i}(t)$ transforms (5.1)-(5.2) into the system (5.6)-(5.7), now considering $P C_{\varepsilon}$ as the phase space, which has the form of (5.8) with the same functions $\bar{a}_{i}, \bar{\beta}_{i}, \bar{f}_{i}$, and $\bar{I}_{i k}$. Similar computations lead to

$$
\left|\bar{f}_{i}(t, \phi)-\bar{f}_{i}(t, \varphi)\right| \leq l_{i}\|\phi-\varphi\|_{\varepsilon}, \quad i=1, \ldots, n,
$$

for all $\phi, \varphi \in P C_{\varepsilon}$ and $t \geq 0$, with $l_{i}:=d_{i}^{-1} \sum_{j=1}^{n} d_{j} \sum_{p=1}^{P}\left(\zeta_{i j}^{(p)} e^{\varepsilon \tau_{i j}^{(p)}}+\mu_{i j}^{(p)} \sigma_{i j}^{(p)} \int_{-\infty}^{0} e^{-\varepsilon s} d \eta_{i j}^{(p)}(s)\right)$.

This means that (A2) holds and from (5.9) the hypothesis (A8) also holds. Now the conclusion follows from Theorem 4.2 .

Remark 5.1. In the previous two theorems, it is specially relevant the situation where $\widehat{\gamma}_{k}>1$ for large $k$ (that is, in Theorem 5.2 the constant $\eta$ is strictly positive), since this allows the solution to be further away from the equilibrium point after the impulsive moments, than before their occurence. Although this situation happens quite often in the real world, it does not fit into the setting of most of the literature. In fact, in many papers [2] , [19], [24], [25], [31], [33], the impulsive operators $I_{i k}$ are defined as

$$
I_{i k}(u)=-\alpha_{i k}\left(u-x_{i}^{*}\right), \quad u \in \mathbb{R}, \text { with } 0<\alpha_{i k}<2,
$$

where $x^{*}=\left(x_{1}^{*}, \ldots, x_{n}^{*}\right) \in \mathbb{R}^{n}$ is the equilibrium point of the neural network model. From (5.10), we conclude that

$$
\left|x_{i}\left(t_{k}^{+}\right)-x_{i}^{*}\right|=\left|1-\alpha_{i k}\right|\left|x_{i}\left(t_{k}\right)-x_{i}^{*}\right|<\left|x_{i}\left(t_{k}\right)-x_{i}^{*}\right|,
$$

for each $k \in \mathbb{N}$ and $i=1, \ldots, n$, which imposes what seems to be (in spite of its applications to some control problems) a very severe and unrealistic constraint: the solutions of the impulsive model must be nearer of the equilibrium point than the solutions of the model without impulses.

On the other hand, this gives rise to an interesting question. First, we note that, in the absence of impulses, the criteria for stability provided by Theorems 4.1 and 4.2 coincide with the ones established in [5], [6], [28]. In many real world situation, one however expects that impulses satisfying (5.10) are introduced to control the behaviour of solutions and force 
them to converge to the equilibrium. This suggests that, if the conditions (5.10) are satisfied, maybe a criterion for the attractivity of the equilibrium point will be achieved under weaker conditions on $f$ than the ones above.

Example 5.1. Consider the following Cohen-Grossberg neural networks model with impulses:

$$
\begin{gathered}
\dot{x}_{i}(t)=-a_{i}\left(x_{i}(t)\right)\left[b_{i}\left(x_{i}(t)\right)-\sum_{j=1}^{n} a_{i j} f_{j}\left(x_{j}(t)\right)-\sum_{j=1}^{n} b_{i j} g_{j}\left(x_{j}\left(t-\tau_{i j}(t)\right)\right)\right. \\
\left.\quad-\sum_{j=1}^{n} c_{i j} h_{j}\left(\int_{-\infty}^{0} K_{i j}(-s) x_{j}(t+s) d s\right)+J_{i}\right], 0 \leq t \neq t_{k}, \\
\Delta\left(x_{i}\left(t_{k}\right)\right)=I_{i k}\left(x_{i}\left(t_{k}^{-}\right)\right), i=1, \ldots n, k \in \mathbb{N},
\end{gathered}
$$

where $a_{i j}, b_{i j}, c_{i j} \in \mathbb{R}, a_{i}: \mathbb{R} \rightarrow(0, \infty), b_{i}: \mathbb{R} \rightarrow \mathbb{R}$, and $\tau_{i j}:[0, \infty) \rightarrow[0, \infty)$ are continuous functions, with $\tau_{i j}(t) \leq \tau_{i j} \leq \tau, f_{j}, g_{j}$, and $h_{j}$ are Lipschitz functions with Lipschiz constants $F_{j}, G_{j}$, and $H_{j}$ respectively, and $K_{i j}$ are nonnegative continuous functions such that

$$
\int_{0}^{\infty} K_{i j}(t) d t=1, \quad i, j=1, \ldots, n .
$$

Since the system (5.11)-(5.12) is a particular case of (5.1)-(5.2), Theorem 5.1 applied to (5.11)-(5.12) gives the following result:

Corollary 5.1. Consider (5.11)-(5.12) under the hypotheses considered above, and, for $i, j=1, \ldots, n$, assume that:

(i) $b_{i}$ satisfy (A1);

(ii) $I_{i k}$ satisfy (A4) and (A5);

(iii) (A6) holds;

(iv) the matrix $N=B-\left[l_{i j}\right]$, where $B=\operatorname{diag}\left(\beta_{1}, \ldots, \beta_{n}\right)$ for $\beta_{i}$ as in (A1) and $l_{i j}=$ $\left|a_{i j}\right| F_{j}+\left|b_{i j}\right| G_{j}+\left|c_{i j}\right| H_{j}$, is a non-singular M-matrix.

Then there is a unique equilibrium point $x^{*}$ of (5.11)-(5.12), which is globally asymptotically stable.

As a consequence of Theorem 5.2, we have the following result:

Corollary 5.2. Consider (5.11)-(5.12) under the hypotheses considered above, and, for $i, j=1, \ldots, n$, assume that:

(i) $b_{i}$ satisfy (A1);

(ii) $I_{\text {ik }}$ satisfy (A4);

(iii) $a_{i}$ satisfy (A7);

(iv) there is $k^{*} \in \mathbb{N}$ such that the conditions

$$
\int_{0}^{\infty} K_{i j}(t) e^{\gamma t} d t<\infty, \quad i, j=1, \ldots, n
$$

hold for some $\gamma>\eta:=\sup _{k \geq k^{*}}\left(\frac{\log \left(\max \left\{1, \widehat{\gamma}_{k}\right\}\right)}{t_{k}-t_{k-1}}\right)$, where $\widehat{\gamma}_{k}:=\max _{1 \leq i \leq n} \widehat{\gamma}_{i k}$.

If the matrix

$$
M=D-\left[n_{i j}\right],
$$


where $D=\operatorname{diag}\left(\beta_{1}-\frac{\eta}{\underline{a_{1}}}, \ldots, \beta_{n}-\frac{\eta}{\underline{a_{n}}}\right)$ and $n_{i j}=\left|a_{i j}\right| F_{j}+\left|b_{i j}\right| e^{\eta \tau_{i j}} G_{j}+\left|c_{i j}\right| H_{j} \int_{-\infty}^{0} K_{i j}(-s) e^{-\eta s} d s$, is a non-singular M-matrix, then there is a unique equilibrium point $x^{*}$ of (5.11)-(5.12), which is globally exponentially stable.

Remark 5.2. System (5.11)-(5.12) was studied in [21]. We note that this system is only a particular case of (5.1)-(5.2), hence our Theorem 5.2 is more general than the main result in $[21]$.

Example 5.2. The following impulsive Cohen-Grossberg-type BAM neural network model is also a particular case of the impulsive model (5.1)-(5.2):

$$
\left\{\begin{array}{l}
\dot{x}_{i}(t)=-a_{i}\left(x_{i}(t)\right)\left[b_{i}\left(x_{i}(t)\right)+\sum_{j=1}^{m}\left(c_{i j} g_{j}\left(y_{j}(t)\right)\right)+\right. \\
\left.\left.\quad+d_{i j} \int_{-\infty}^{0} K_{i j}(-s) g_{j}\left(y_{j}(t+s)\right) d s\right)+J_{i}\right], 0 \leq t \neq t_{k}, \\
\Delta\left(x_{i}\left(t_{k}\right)\right)=I_{i k}\left(x_{i}\left(t_{k}^{-}\right)\right), i=1, \ldots n, k \in \mathbb{N} ; \\
\dot{y}_{j}(t)=-\bar{a}_{j}\left(y_{j}(t)\right)\left[\bar{b}_{j}\left(y_{j}(t)\right)+\sum_{i=1}^{n}\left(\bar{c}_{j i} f_{i}\left(x_{i}(t)\right)\right)+\right. \\
\left.\left.\quad+\bar{d}_{j i} \int_{-\infty}^{0} \bar{K}_{j i}(-s) f_{i}\left(x_{i}(t+s)\right) d s\right)+\bar{J}_{j}\right], 0 \leq t \neq t_{k}, \\
\Delta\left(y_{j}\left(t_{k}\right)\right)=\bar{I}_{j k}\left(y_{j}\left(t_{k}^{-}\right)\right), j=1, \ldots m, k \in \mathbb{N}
\end{array}\right.
$$

where $t_{k} \nearrow \infty$ as $k \rightarrow \infty, c_{i j}, \bar{c}_{j i}, d_{i j}, \bar{d}_{j i} \in \mathbb{R}, a_{i}, \bar{a}_{j}: \mathbb{R} \rightarrow(0, \infty), b_{i}, \bar{b}_{j}, I_{i k}, \bar{I}_{j k}: \mathbb{R} \rightarrow \mathbb{R}$, are continuous functions, $g_{j}, f_{i}: \mathbb{R} \rightarrow \mathbb{R}$ are Lipschitz functions with Lipschiz constants $G_{j}, F_{i}$, respectively, and $K_{i j}, \bar{K}_{j i}:[0, \infty) \rightarrow[0, \infty)$ are nonnegative continuous functions such that

$$
\int_{0}^{\infty} K_{i j}(t) d t=\int_{0}^{\infty} \bar{K}_{j i}(t) d t=1
$$

for all $i \in\{1, \ldots, n\}, j \in\{1, \ldots, m\}$.

Corollary 5.3. For the above system (5.13), for $i=1, \ldots, n, j=1, \ldots, m$ assume that:

(i) $b_{i}, \bar{b}_{j}$ satisfy (A1) with constants $\beta_{i}, \bar{\beta}_{j}$, respectively;

(ii) $I_{i k}, \bar{I}_{j k}$ satisfy (A4) with constants $\widehat{\gamma}_{i k}, \widehat{\bar{\gamma}}_{j k}$, respectively;

(iii) for $\widehat{\gamma}_{k}:=\max _{1 \leq i \leq n, 1 \leq j \leq m}\left\{\widehat{\gamma}_{i k}, \widehat{\bar{\gamma}}_{j k}\right\}, \prod_{k=1}^{\infty} \max \left\{1, \widehat{\gamma}_{k}\right\}<\infty$;

(iv) (A6) holds;

(v) the matrix

$$
N=\left(\begin{array}{cc}
B & 0 \\
0 & \bar{B}
\end{array}\right)_{(n+m) \times(n+m)}-\left(\begin{array}{cc}
0 & (|C|+|D|) G \\
(|\bar{C}|+|\bar{D}|) F & 0
\end{array}\right)_{(n+m) \times(n+m)}
$$

where $B=\operatorname{diag}\left(\beta_{1}, \ldots, \beta_{n}\right), \bar{B}=\operatorname{diag}\left(\bar{\beta}_{1}, \ldots, \bar{\beta}_{m}\right)$ for $\beta_{i}, \bar{\beta}_{j}$ as in $(A 1)$ and $|C|=\left[\left|c_{i j}\right|\right]_{n \times m}$, $|\bar{C}|=\left[\left|\bar{c}_{j i}\right|\right]_{m \times n},|D|=\left[\left|d_{i j}\right|\right]_{n \times m},|\bar{D}|=\left[\left|\bar{d}_{j i}\right|\right]_{m \times n}, F=\operatorname{diag}\left(F_{1}, \ldots, F_{n}\right)$, and $G=$ $\operatorname{diag}\left(G_{1}, \ldots, G_{m}\right)$, is a non-singular M-matrix.

Then there is a unique equilibrium point $\left(x_{1}^{*}, \ldots, x_{n}^{*}, y_{1}^{*}, \ldots, y_{m}^{*}\right) \in \mathbb{R}^{n+m}$ of (5.13), which is globally asymptotically stable. 
The exponential stability of the equilibrium point of (5.13) was recently studied in [22], with the $p$-norm in $\mathbb{R}^{n}, p \geq 1$. (The case $p=\infty$ was not treated in [22]). As a consequence of Theorem 5.2, we have the following stability criterion with the $\infty$-norm, which complements the result in [22].

Corollary 5.4. For the above system (5.13), for $i=1, \ldots, n, j=1, \ldots, m$ assume that:

(i) $b_{i}, \bar{b}_{j}$ satisfy (A1) with constants $\beta_{i}, \bar{\beta}_{j}$, respectively;

(ii) $I_{i k}, \bar{I}_{j k}$ satisfy (A4) with constants $\widehat{\gamma}_{i k}, \widehat{\bar{\gamma}}_{j k}$, respectively;

(iii) $a_{i}, \bar{a}_{j}$ satisfy (A7) with constants $\underline{a_{i}}, \underline{\bar{a}_{j}}$, respectively;

(iv) there is $k^{*} \in \mathbb{N}$ such that

$$
\int_{0}^{\infty} K_{i j}(t) e^{\gamma t} d t<\infty, \quad \int_{0}^{\infty} \bar{K}_{j i}(t) e^{\gamma t} d t<\infty
$$

hold for some $\gamma>\eta:=\sup _{k \geq k^{*}}\left(\frac{\log \left(\max _{i, j}\left\{1, \widehat{\gamma}_{i k}, \widehat{\bar{\gamma}}_{j k}\right\}\right)}{t_{k}-t_{k-1}}\right)$.

If the matrix

$$
M=\left(\begin{array}{cc}
B-E & 0 \\
0 & \bar{B}-\bar{E}
\end{array}\right)_{(n+m) \times(n+m)}-\left(\begin{array}{cc}
0 & \left(|C|+\left|D_{K}\right|\right) G \\
\left(|\bar{C}|+\left|\bar{D}_{K}\right|\right) F & 0
\end{array}\right)_{(n+m) \times(n+m)}
$$

with $B, \bar{B},|C|, F, G$ defined as in the above corollary, $E=\operatorname{diag}\left(\frac{\eta}{\underline{a_{1}}}, \ldots, \frac{\eta}{a_{n}}\right), \bar{E}=$ $\operatorname{diag}\left(\frac{\eta}{\underline{\bar{a}_{1}}}, \ldots, \frac{\eta}{\underline{\bar{a}_{m}}}\right),\left|D_{K}\right|=\left[\left|d_{i j}\right| \int_{-\infty}^{0} K_{i j}(-s) e^{-\eta s} d s\right]_{n \times m},\left|\bar{D}_{K}\right|=\left[\left|\bar{d}_{j i}\right| \int_{-\infty}^{0} \bar{K}_{j i}(-s) e^{-\eta s} d s\right]_{m \times n}$, is a non-singular M-matrix, then there is a unique equilibrium point $\left(x_{1}^{*}, \ldots, x_{n}^{*}, y_{1}^{*}, \ldots, y_{m}^{*}\right) \in$ $\mathbb{R}^{n+m}$ of (5.13), which is globally exponentially stable.

Example 5.3. In [33], the following BAM neural network model with impulses was considered:

$$
\left\{\begin{array}{l}
\dot{x}_{i}(t)=-b_{i} x_{i}(t)+\sum_{j=1}^{m}\left(c_{i j} g_{j}\left(y_{j}(t)\right)+d_{i j} g_{j}\left(y_{j}\left(t-\tau_{i j}\right)\right)\right)+J_{i}, 0 \leq t \neq t_{k} \\
\Delta\left(x_{i}\left(t_{k}\right)\right)=I_{i k}\left(x_{i}\left(t_{k}^{-}\right)\right), i=1, \ldots n, k \in \mathbb{N} \\
\dot{y}_{j}(t)=-\bar{b}_{j} y_{j}(t)+\sum_{i=1}^{n}\left(\bar{c}_{j i} f_{i}\left(x_{i}(t)\right)+\bar{d}_{j i} f_{i}\left(x_{i}\left(t-\sigma_{j i}\right)\right)\right)+\bar{J}_{j}, 0 \leq t \neq t_{k} \\
\Delta\left(y_{j}\left(t_{k}\right)\right)=\bar{I}_{j k}\left(y_{j}\left(t_{k}^{-}\right)\right), j=1, \ldots m, k \in \mathbb{N}
\end{array}\right.
$$

where $b_{i}, \bar{b}_{j} \in(0, \infty), c_{i j}, \bar{c}_{j i}, d_{i j}, \bar{d}_{j i}, J_{i}, \bar{J}_{j} \in \mathbb{R}, \tau_{i j}, \sigma_{j i} \in[0, \infty), I_{i k}, \bar{I}_{j k}: \mathbb{R} \rightarrow \mathbb{R}$ are continuous functions and $f_{i}, g_{j}: \mathbb{R} \rightarrow \mathbb{R}$ are Lipschitz functions with Lipschitz constants $F_{i}, G_{j}$, respectively, $i=1, \ldots, n, j=1, \ldots, m$.

Clearly, system (5.14) is still a particular case of (5.1)-(5.2), and from Theorems 5.1 and 5.2 we obtain the next two results.

Corollary 5.5. Consider (5.14) under the hypotheses above, and, for $i=1, \ldots, n, j=$ $1, \ldots, m$, and $k \in \mathbb{N}$ assume that:

(i) $I_{i k}, \bar{I}_{j k}$ satisfy (A4) with constants $\widehat{\gamma}_{i k}, \widehat{\bar{\gamma}}_{j k}$, respectively; 
(ii) for $\widehat{\gamma}_{k}:=\max _{1 \leq i \leq n, 1 \leq j \leq m}\left\{\widehat{\gamma}_{i k}, \widehat{\bar{\gamma}}_{j k}\right\}, \prod_{k=1}^{\infty} \max \left\{1, \widehat{\gamma}_{k}\right\}<\infty$;

(iii) (A6) holds;

(iv) the matrix

$$
N=\left(\begin{array}{cc}
B & 0 \\
0 & \bar{B}
\end{array}\right)_{(n+m) \times(n+m)}-\left(\begin{array}{cc}
0 & (|C|+|D|) G \\
(|\bar{C}|+|\bar{D}|) F & 0
\end{array}\right)_{(n+m) \times(n+m)}
$$

where $B=\operatorname{diag}\left(b_{1}, \ldots, b_{n}\right), \bar{B}=\operatorname{diag}\left(\bar{b}_{1}, \ldots, \bar{b}_{m}\right),|C|=\left[\left|c_{i j}\right|\right]_{n \times m},|\bar{C}|=\left[\left|\bar{c}_{j i}\right|\right]_{m \times n},|D|=$ $\left[\left|d_{i j}\right|\right]_{n \times m},|\bar{D}|=\left[\left|\bar{d}_{j i}\right|\right]_{m \times n}, F=\operatorname{diag}\left(F_{1}, \ldots, F_{n}\right)$, and $G=\operatorname{diag}\left(G_{1}, \ldots, G_{m}\right)$, is a nonsingular M-matrix.

Then there is a unique equilibrium point $\left(x_{1}^{*}, \ldots, x_{n}^{*}, y_{1}^{*}, \ldots, y_{m}^{*}\right) \in \mathbb{R}^{n+m}$ of (5.14), which is globally asymptotically stable.

Corollary 5.6. Consider (5.14) under the hypotheses above, and, for $i=1, \ldots, n, j=$ $1, \ldots, m$, and $k \in \mathbb{N}$ assume that:

(i) $I_{i k}, \bar{I}_{j k}$ satisfy (A4) with constants $\widehat{\gamma}_{i k}, \widehat{\bar{\gamma}}_{j k}$, respectively.

(ii) For $\eta=\sup _{k \geq k^{*}}\left(\frac{\log \left(\max _{i, j}\left\{1, \gamma_{i k}, \bar{\gamma}_{j k}\right\}\right)}{t_{k}-t_{k-1}}\right)$, the matrix

$$
M=\left(\begin{array}{cc}
B & 0 \\
0 & \bar{B}
\end{array}\right)-\left(\begin{array}{cc}
E & \left(|C|+\left|D_{\eta}\right|\right) G \\
\left(|\bar{C}|+\left|\bar{D}_{\eta}\right|\right) F & \bar{E}
\end{array}\right)
$$

where $B, \bar{B},|C|, F, G$ are as in the above result, and $E=\operatorname{diag}(\eta, \ldots, \eta)_{n \times n}, \bar{E}=$ $\operatorname{diag}(\eta, \ldots, \eta)_{m \times m},\left|D_{\eta}\right|=\left[\left|d_{i j}\right| e^{\eta \tau_{i j}}\right]_{n \times m},\left|\bar{D}_{\eta}\right|=\left[\left|\bar{d}_{j i}\right| e^{\eta \sigma_{j i}}\right]_{m \times n}$, is a non-singular $M$ matrix.

Then there is a unique equilibrium point $\left(x_{1}^{*}, \ldots, x_{n}^{*}, y_{1}^{*}, \ldots, y_{m}^{*}\right) \in \mathbb{R}^{n+m}$ of (5.14), which is globally exponentially stable.

Remark 5.3. In [33], the impulsive operators $I_{i k}, \bar{I}_{j k}$ are defined as

$$
\begin{array}{ll}
I_{i k}(u)=-\alpha_{i k}\left(u-x_{i}^{*}\right), & \text { with } 0<\alpha_{i k}<2, \\
\bar{I}_{j k}(u)=-\bar{\alpha}_{j k}\left(u-y_{j}^{*}\right), & \text { with } 0<\bar{\alpha}_{j k}<2,
\end{array}
$$

where $\left(x_{1}^{*}, \ldots, x_{n}^{*}, y_{1}^{*}, \ldots, y_{m}^{*}\right) \in \mathbb{R}^{n+m}$ is the equilibrium point of (5.14). Consequently, $\widehat{I}_{i k}(u)=\left(1-\alpha_{i k}\right) u-\alpha_{i k} x_{i}^{*}$ and $\widehat{\bar{I}}_{j k}(u)=\left(1-\bar{\alpha}_{j k}\right) u-\bar{\alpha}_{j k} y_{j}^{*}$ and therefore (A4) holds with $\gamma_{i k}=\left|1-\alpha_{i k}\right|<1$ and $\bar{\gamma}_{j k}=\left|1-\bar{\alpha}_{j k}\right|<1$ and $\eta=0$. Then we conclude that Corollary 5.6 strongly improves the main result in [33].

\section{Acknowledgements}

This research was supported by Fundação para a Ciência e a Tecnologia, Financiamento Base 2010 ISFL-1-209 (T. Faria) and through the research center CMAT (J.J. Oliveira), and by FAPESP - Processo 2010/12712-7 (M. Gadotti). This work was partially written while M. Gadotti was visiting the research center CMAF of the University of Lisbon, and she thanks the center for its kind hospitality. T. Faria thanks Prof. Valeri Obukhovskii for a fruitful discussion, which has lead to an improvement in Section 3. 


\section{References}

[1] G. Ballinger and X. Liu, Existence, uniqueness and boundedness results for impulsive delay differential equations, Applicable Anal. 74 (2000), 71-93.

[2] J. Chen and B. Cui, Impulsive effects on global asymptotic stability of delay BAM neural networks, Chaos Solutions \& Fractals, 38 (2008) 1115-1125.

[3] J. Dieudonné, Éléments d'Analyse. Tome I: Fondements de L'Analyse Moderne, Gauthieur-Villars, Paris, 1969.

[4] N. Dunford and J. T. Schwartz, Linear operators, Part I, Wiley-Interscience, New York, 1966.

[5] T. Faria and J.J. Oliveira, Boundedness and global exponential stability for delayed differential equations with applications, Appl. Math. Comput., 214 (2009) 487-496.

[6] T. Faria and J.J. Oliveira, General criteria for asymptotic and exponential stabilities of neural network models with unbounded delays, Appl. Math. Comput., 217 (2011) 9646-9658.

[7] M. Fiedler, Special Matrices and Their Applications in Numerical Mathematics, Martinus Nijhoff Publ. (Kluwer), Dordrecht, 1986.

[8] X. L. Fu and L. Zhou, Stability for Impulsive Functional Differential Equations with Infinite Delays, Acta Mathematica Sinica, English Series, 26 (2010) 909-922.

[9] L.P. Gimenes, M. Federson and P. Táboas, Impulsive stability for systems of second order retarded differential equations, Nonlinear Anal. 67 (2007), 545-553.

[10] K. Gopalsamy, Stability of artificial neural networks with impulses, Appl. Math. Comput., 154 (2004), 783-813.

[11] A. Granas and J. Dugundji, Fixed Point Theory, Springer-Verlag, New York, 2003.

[12] J. R. Haddock and W. E. Hornor, Precompactness and convergence in norm of positive orbits in a certain fading memory space, Funkcial. Ekvac. 31 (1988), 349-361.

[13] J.K.Hale and J. Kato, Phase space for retarded equations with infinite delay, Funkcial. Ekvac. 21 (1978), 11-41.

[14] J.K. Hale and S.M. Verduyn Lunel, Introduction to Functional Differential Equations, Springer-Verlag, New York (1993).

[15] E. Hernández, M. Rabello and H.R. Henríquez, Existence of solutions for impulsive partial neutral functional differential equations, J. Math. Anal. appl. 331 (2007), 11351158 .

[16] Y. Hino, S. Murakami, T. Naito, Functional Differential Equations with Infinite Delay, Springer-Verlag, Berlin (1991).

[17] C. S. Hönig, Volterra Stieltjes-Integral Equations, North-Holland Publ. Co., 16 (1975), New York. 
[18] J.J. Hopfield, Neural networks and physical systems with emergent collective computational abilities, Proc. Nat. Acad. Sci. U.S.A. 79 (1982), 2554-2558.

[19] Z.T. Huang, X.S. Luo and Q.G. Yang, Global asymptotic stability analysis of bidirectional associative memory neural network with distributed delays and impulse, Chaos Solutions \& Fractals, 34 (2007) 878-885.

[20] V. Lakshimikantham, D.D. Bainov and P.S. Siomeonov, Theory of Impulsive Differential Equations, Singarope: World Scientific (1989).

[21] K. Li, Stability analysis for impulsive Cohen-Grossberg neural networks with time-varyng delays and distributed delays, Nonlinear Anal. RWA 10 (2009) 2784-2798.

[22] K. Li, L. Zhang, X. Zhang, Z. Li, Stability in impulsive Cohen-Grossberg-type BAM neural networks with distributed delays, Appl. Math. Comput. 215 (2010) 3970-3984.

[23] X. Liu and G. Ballinger, Existence and continuity of solutions for differential equations with delays and state-dependent impulses, Nonlinear Anal. 51 (2002), 633-647.

[24] X.Y. Luo and B.T. Cui, Global asymptotic stability of delay BAM neural networks with impulses, Chaos, Solitons and Fractals, 29(2006) 1023-1031.

[25] W. Luo, S. Zhong and J. Yang, Global exponential stability of impulsive Cohen-Grossberg neural networks with delays, Chaos, Solitons and Fractals, 42 (2009) 1084-1091.

[26] Z. Luo and J. Shen, Stability results for impulsive functional differential equations with infinite delays, J. Comput. Appl. Math. 131 (2001) 55-64.

[27] Z. Luo and J. Shen, Stability and boundedness for impulsive functional differential equations with infinite delay, Nonlinear Anal. 46 (2001), 475-493.

[28] J.J. Oliveira, Global stability of a Cohen-Grossberg neural network with both timevarying and continuous distributed delays, Nonlinear Anal. RWA, 12 (2011) 2861-2870.

[29] A. Ouahab, Local and global existence and uniqueness results for impulsive functional differential equations with multiple delay, J. Math. Anal. Appl. 323 (2006), 456-472.

[30] A. Ouahab, Existence and uniqueness results for impulsive functional differential equations with scalar multiple delay and infinite delay, Nonlinear Anal., 67 (2007), 1027-1041.

[31] R. Samidurai, R. Sakthivel and S.M. Anthoni, Global asymptotic stability of BAM neural networks with mixed delays and impulses, Mathematical Metholds in the Applied Sciences 33 (2010) 2107-2117.

[32] G. Sun and X. Li, A new criterion to global exponential stability for impulsive neural networks with continuously distributed delays, Math. Methods Appl. Sciences 33 (2010) 2107-2117.

[33] Y. Xia, Z. Huang, M. Han, Existence and globally exponential stability of equilibrium for BAM neural networks with impulses, Chaos Solutions \& Fractals, 37 (2008) 588-597.

[34] R. Ye, Existence of solutions for impulsive partial neutral functional differential equation with infinite delay, Nonlinear Anal. 73 (2010), 155-162. 